\title{
\begin{tabular}{l|l} 
Mitraries & DSpace@MIT
\end{tabular}
}

\author{
MIT Open Access Articles
}

\section{M-DWARF RAPID ROTATORS AND THE DETECTION OF RELATIVELY YOUNG MULTIPLE M-STAR SYSTEMS}

The MIT Faculty has made this article openly available. Please share how this access benefits you. Your story matters.

Citation: Rappaport, S., J. Swift, A. Levine, M. Joss, R. Sanchis-Ojeda, T. Barclay, M. Still, et al. "M-DWARF RAPID ROTATORS AND THE DETECTION OF RELATIVELY YOUNG MULTIPLE MSTAR SYSTEMS." The Astrophysical Journal 788, no. 2 (May 29, 2014): 114. (C) 2014 The American Astronomical Society

As Published: http://dx.doi.org/10.1088/0004-637x/788/2/114

Publisher: IOP Publishing

Persistent URL: http://hdl.handle.net/1721.1/93142

Version: Final published version: final published article, as it appeared in a journal, conference proceedings, or other formally published context

Terms of Use: Article is made available in accordance with the publisher's policy and may be subject to US copyright law. Please refer to the publisher's site for terms of use. 


\title{
M-DWARF RAPID ROTATORS AND THE DETECTION OF RELATIVELY YOUNG MULTIPLE M-STAR SYSTEMS
}

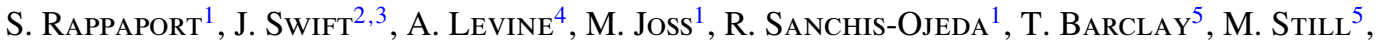

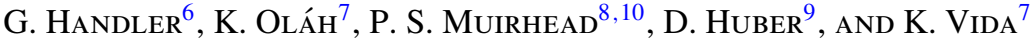 \\ ${ }^{1}$ Department of Physics, Kavli Institute for Astrophysics and Space Research, Massachusetts Institute of Technology, Cambridge, MA 02139, USA; \\ sar@mit.edu,mattjoss@mit.edu, rsanchis86@gmail.com \\ 2 Department of Astronomy, California Institute of Technology, MC 249-17, Pasadena, CA 91125, USA; jswift@ astro.caltech.edu \\ ${ }^{3}$ Department of Planetary Science, California Institute of Technology, MC 249-17, Pasadena, CA 91125, USA; jswift@astro.caltech.edu \\ ${ }^{4}$ 37-575 M.I.T. Kavli Institute for Astrophysics and Space Research, 70 Vassar St., Cambridge, MA 02139, USA; aml@ space.mit.edu \\ ${ }^{5}$ BAER Institute/NASA Ames Research Center, M/S 244-30, Moffett Field, Mountain View, CA 94035, USA; thomas.barclay@ nasa.gov, martin.d.still@nasa.gov \\ ${ }^{6}$ Nicolaus Copernicus Astronomical Center, Bartycka 18, PL 00-716 Warsaw, Poland; gerald@camk.edu.pl \\ ${ }^{7}$ Konkoly Observatory, MTA CSFK, Konkoly Thege Miklós út 15-17 H-1525 Budapest, Hungary; olah@konkoly.hu, vidakris@konkoly.hu \\ ${ }^{8}$ Department of Astronomy, Boston University, Boston, MA 02215, USA; philipm@bu.edu \\ ${ }^{9}$ SETI Institute/NASA Ames Research Center, MS 244-30, Moffett Field, CA 94035, USA; daniel.huber@nasa.gov \\ Received 2014 February 13; accepted 2014 April 30; published 2014 May 29
}

\begin{abstract}
We have searched the Kepler light curves of $\sim 3900$ M-star targets for evidence of periodicities that indicate, by means of the effects of starspots, rapid stellar rotation. Several analysis techniques, including Fourier transforms, inspection of folded light curves, "sonograms," and phase tracking of individual modulation cycles, were applied in order to distinguish the periodicities due to rapid rotation from those due to stellar pulsations, eclipsing binaries, or transiting planets. We find 178 Kepler M-star targets with rotation periods, $P_{\text {rot }}$, of $<2$ days, and 110 with $P_{\text {rot }}<1$ day. Some 30 of the 178 systems exhibit two or more independent short periods within the same Kepler photometric aperture, while several have 3 or more short periods. Adaptive optics imaging and modeling of the Kepler pixel response function for a subset of our sample support the conclusion that the targets with multiple periods are highly likely to be relatively young physical binary, triple, and even quadruple $\mathrm{M}$ star systems. We explore in detail the one object with four incommensurate periods all less than 1.2 days, and show that two of the periods arise from one of a close pair of stars, while the other two arise from the second star, which itself is probably a visual binary. If most of these M-star systems with multiple periods turn out to be bound M stars, this could prove a valuable way discovering young hierarchical M-star systems; the same approach may also be applicable to $\mathrm{G}$ and $\mathrm{K}$ stars. The $\sim 5 \%$ occurrence rate of rapid rotation among the $\sim 3900 \mathrm{M}$ star targets is consistent with spin evolution models that include an initial contraction phase followed by magnetic braking, wherein a typical $\mathrm{M}$ star can spend several hundred Myr before spinning down to periods longer than 2 days.
\end{abstract}

Key words: binaries: close - binaries: general - stars: activity - stars: late-type - stars: rotation - starspots techniques: photometric

Online-only material: color figures

\section{INTRODUCTION}

M dwarfs-main-sequence stars with masses $M_{\star} \lesssim$ $0.6 M_{\odot}$-dominate the Galactic stellar population in number and mass (Chabrier 2003). This preponderance is reflected in the local neighborhood where $\sim 75 \%$ of the nearest stars fall within this spectral class (Henry et al. 1994, 2006; Reid et al. 2002; Cruz \& Reid 2002), and this fraction will continue to grow as more nearby red dwarfs are revealed. Despite their ubiquity, these small stars are difficult to characterize due in part to their low luminosities and complex atmospheres, and models are unable to satisfactorily account for their radii and temperatures at given masses (Torres et al. 2010; Torres \& Ribas 2002; Boyajian et al. 2012; Birkby et al. 2012). The motivation to understand the fundamental properties of $\mathrm{M}$ dwarfs, including their multiplicity, has been amplified by recent results indicating that they frequently host low-mass planets (Swift et al. 2013; Dressing \& Charbonneau 2013; Morton \& Swift 2013).

Low-mass stars lose angular momentum through rotational magnetic braking (Mestel 1968). However, during their premain sequence lifetime their rotation rates increase due to

\footnotetext{
${ }^{10}$ Hubble Postdoctoral Fellow.
}

gravitational contraction. This pre-main sequence phase can last from $\sim 100 \mathrm{Myr}$ for stars of mass $0.5 M_{\odot}$ to several hundred $\mathrm{Myr}$ for the lowest mass stars (see, e.g., Baraffe et al. 2002). After hydrogen burning commences in their cores and gravitational contraction ceases these stars begin to gradually spin down. Consequently, their magnetic field strengths and activity levels tend to decline (Skumanich 1972).

M dwarfs are thought to rotate differentially, but less so than solar-type stars. Recent measurements based on Kepler data show that differential rotation rates for $\mathrm{M}$ dwarfs are typically less than $0.1 \mathrm{rad} \mathrm{day}^{-1}$ (Reinhold et al. 2013). Observations of late-type stars show that their differential rotation rates generally depend strongly on effective temperature and weakly on rotation period (Barnes et al. 2005; Reiners 2006), a behavior that is also seen in computer models (Küker \& Rüdiger 2011).

The Kepler Space Mission (Borucki et al. 2010; Koch et al. 2010) monitored over 150,000 stars nearly continuously for about 4 years, primarily to search for exoplanets (Borucki et al. 2011; Batalha et al. 2013; Burke et al. 2014). Kepler's unprecedented photometric precision has also led to extensive ancillary investigations. ${ }^{11}$

\footnotetext{
${ }^{11}$ keplerscience.arc.nasa.gov/PublicationsAstrophysics.shtml
} 
Though the vast majority of Kepler target stars are Sun-like $\left(0.8 \lesssim M_{\star} \lesssim 1.2 M_{\odot}\right)$, several thousand $\mathrm{M}$ dwarfs have been monitored as well over the course of the primary mission. The initial estimation of the important characteristics of the stars in the Kepler field was done using ground-based multicolor photometry as part of the construction of the Kepler Input Catalog (KIC; Brown et al. 2011). This estimation process was optimized for Sun-like stars; the characteristics of the M dwarfs in the KIC were not determined as accurately and should be adopted only with caution. There have been several efforts to revise the stellar parameters for this M-star sample (e.g., Muirhead et al. 2012; Mann et al. 2012, 2013). In particular, Dressing \& Charbonneau (2013, hereafter DC13) have tabulated and calibrated the properties of 3897 cool and high surface gravity Kepler target stars. The vast majority of these have masses in the range $0.3<M_{\star}<0.6 M_{\odot}$ and the bulk of the effective temperatures fall in the range $3300<T_{\text {eff }}<4000 \mathrm{~K}$. The numbers of stars show a bias toward the higher mass M dwarfs due to the magnitude limited nature of the Kepler targets (Batalha et al. 2010). Some $90 \%$ of the DC13 collection of $\mathrm{M}$ stars are at distances in the range 15-400 pc (median distance of $210 \mathrm{pc}$ ), and have absolute visual magnitudes in the range +8 to +12 .

McQuillan et al. (2013) performed both autocorrelation function ("ACF") and Fourier transform (FT) analyses of the Kepler photometric data for the M dwarf targets in order to identify variations of the stellar fluxes due to starspots and to measure the corresponding periods. The period distribution that they derived is shown in the top panel of Figure 1. The distribution includes periods all the way down to less than a day, has local maxima near 19 days and 33 days, and tails off dramatically toward periods above 40 days. There is also what appears to be a distinct group of $\mathrm{M}$ stars with periods less than $\sim 6$ days, and the distribution is rising toward the shortest periods. Some 55 of these M stars have rotation periods of $\lesssim 2$ days.

In this work we focus on the rapidly rotating $M$ stars, in particular those with $P_{\text {rot }}<1$ day. In Section 2 we describe our search through the Kepler photometric data base for rapidly rotating $M$ stars using an FT algorithm. In Section 3 the possibility that some of the periodicities we observe are due to stellar pulsations is discussed and largely discounted. We show selected examples of "sonograms" for several of our candidate rotators in Section 4, and demonstrate that various frequencies and their harmonics appear to vary independently, thereby arguing for the rotating spotted star hypothesis. In Section 5 we present an analysis where we track the phases of individual starspot rotation cycles and thereby demonstrate that these are not stellar pulsations. In Section 6 we discuss the Kepler M-star targets wherein we have found two or more distinct rotation periods. This includes some 30 with at least two short periods, several with three periods, and one with four distinct periods (all shorter than 1.2 days). We argue in Section 7 that these are actually binary, triple, and quadruply bound M-star systems. In Section 8, we present evidence, based on United Kingdom Infrared Telescope (UKIRT) $J$-band and Keck adaptive optics (AO) images, that these are indeed binary and/or hierarchical systems. In Section 9 we apply a pointspread-function (PSF) analysis to the Kepler pixel-level data for KIC 4660255 which exhibits four short periodicities, and show that two of them arise in each of two stellar images separated by about $4^{\prime \prime}$. We summarize our results and draw some final conclusions in Section 10.
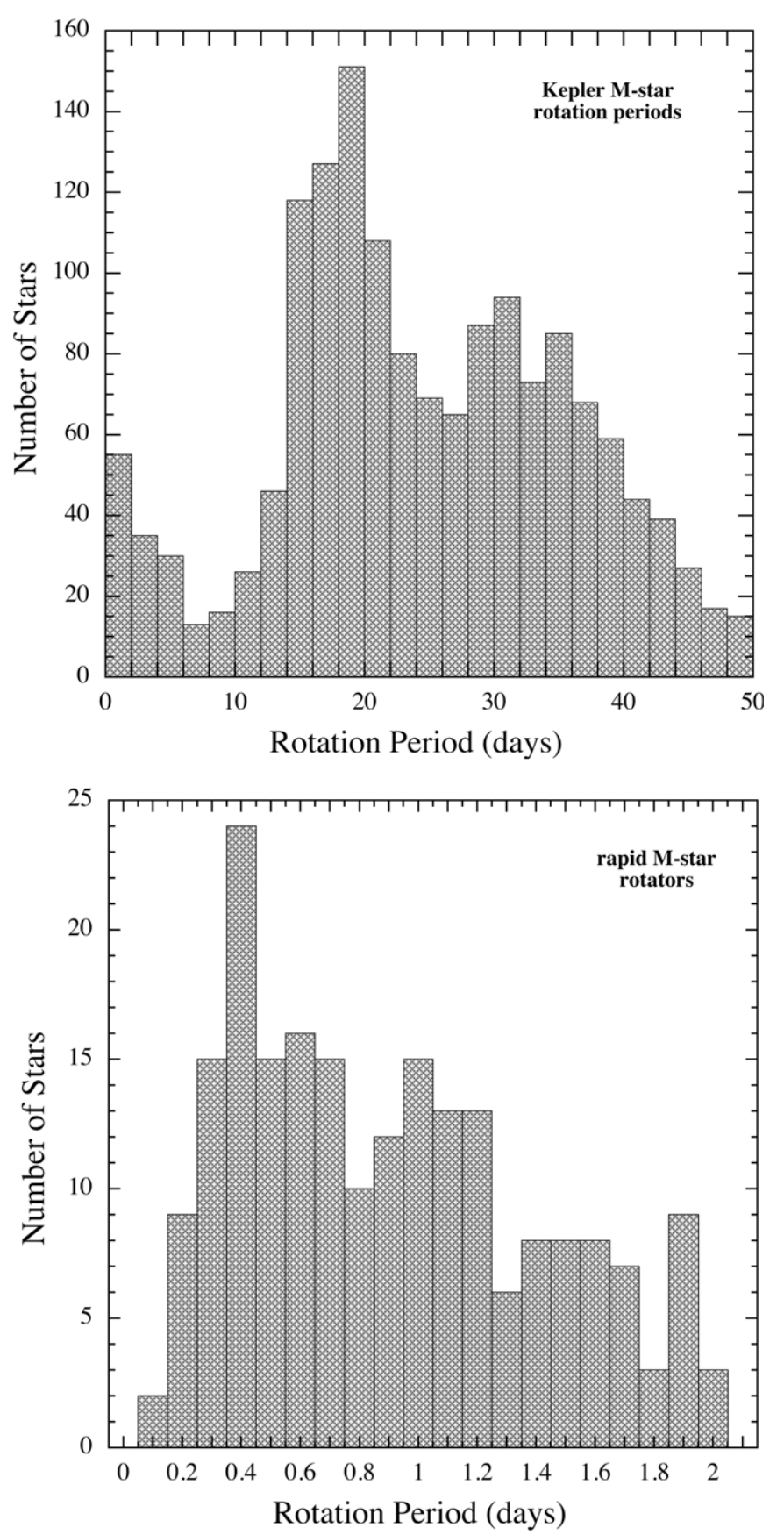

Figure 1. Top: rotation period distribution of $\mathrm{M}$ stars in the Kepler data set; the periods were taken from McQuillan et al. (2013), and are based on 10 months of data. Note the distinct group of relatively rapid rotators with periods shorter than $\sim 5$ days. Bottom: distribution of very short rotation periods found in Kepler M-star targets via our Fourier transform search of all 16 quarters of data. In the case of multiple periods in a single Kepler target, we include all the periods in this histogram.

\section{SEARCH FOR RAPIDLY ROTATING M STARS}

Because the "FT" is an efficient tool for searching for periodic signals with high-duty cycle and smoothly varying profiles (i.e., where the first few harmonics dominate), it was our choice for discovering spotted stars rotating with short periods. While the transitory nature of starspots, which can induce possible erratic changes in the modulation phase, and surface differential rotation can broaden the peaks in a Fourier power spectrum, we did not find it necessary, nor particularly useful, to employ an 

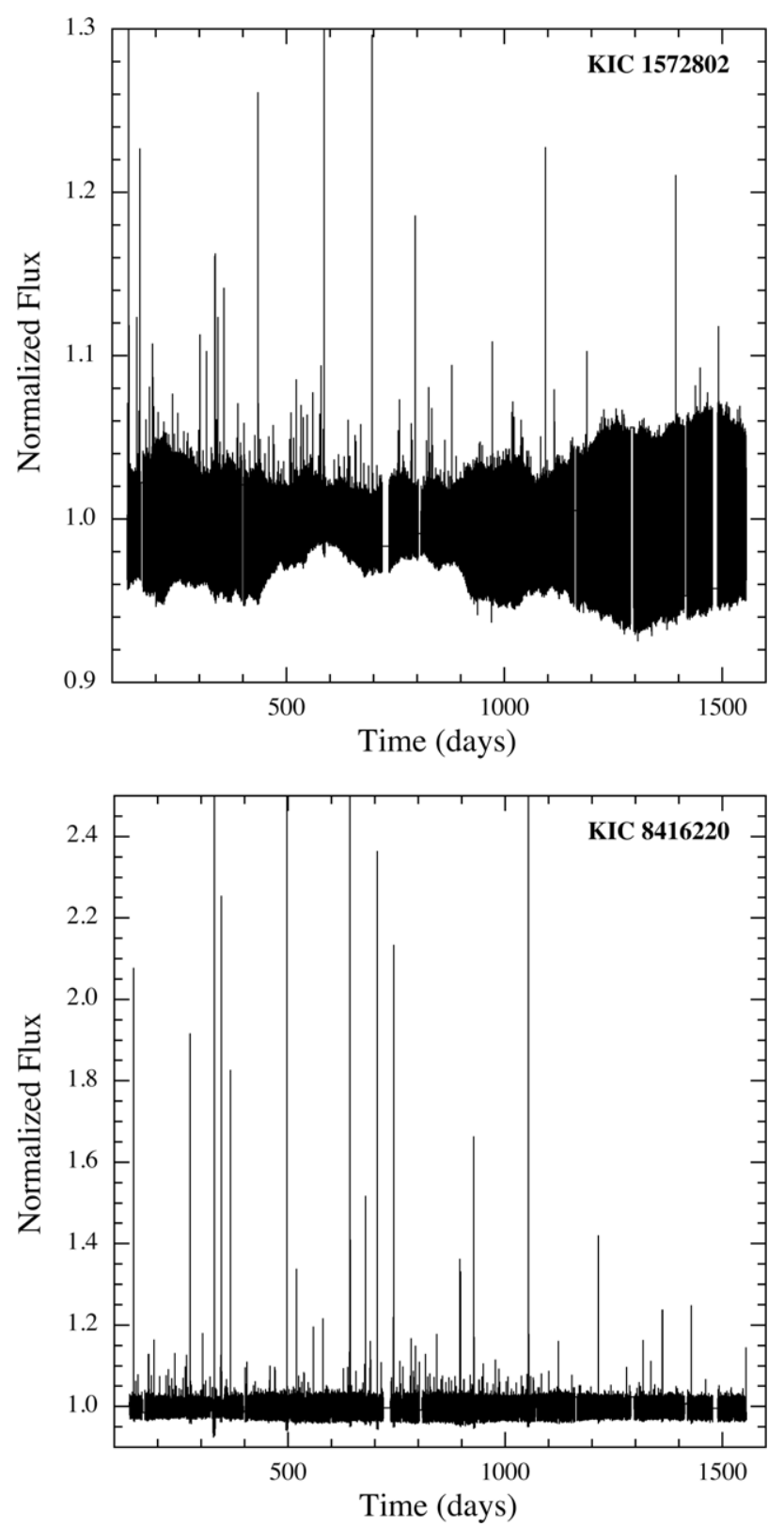

Figure 2. Illustrative Kepler light curves of M-stars. Top: KIC 1572802 which exhibits flares as large as $\sim 30 \%$ of the mean stellar flux, and one periodicity of 0.37 day. Bottom: KIC 8416220 , which has two periods of 0.72 and 0.57 day, and exhibits flares that increase the flux by a whole magnitude.

ACF analysis. As it turns out, we have discovered a substantial number of Kepler M-star targets that exhibit more than one independent period-sometimes close to or near multiples of one another-and the FT is substantially more straightforward than an ACF to use in identifying multiple periods.

The FT approach utilized in this work is very similar in nature to the one used by Sanchis-Ojeda et al. (2013) in their search for short-period planets, and is based on the same selection criteria. In brief, the available Kepler PDCSAP_FLUX data (corrected with PDCMAP; Stumpe et al. 2012; Smith et al. 2012) were normalized quarter-by-quarter with the quarterly median values and then stitched together into a single data file, with all data gaps filled with the mean flux. After the FTs were computed, amplitude spectra were produced with units of parts per million (ppm) variation with respect to a flux of unity. We refer to these
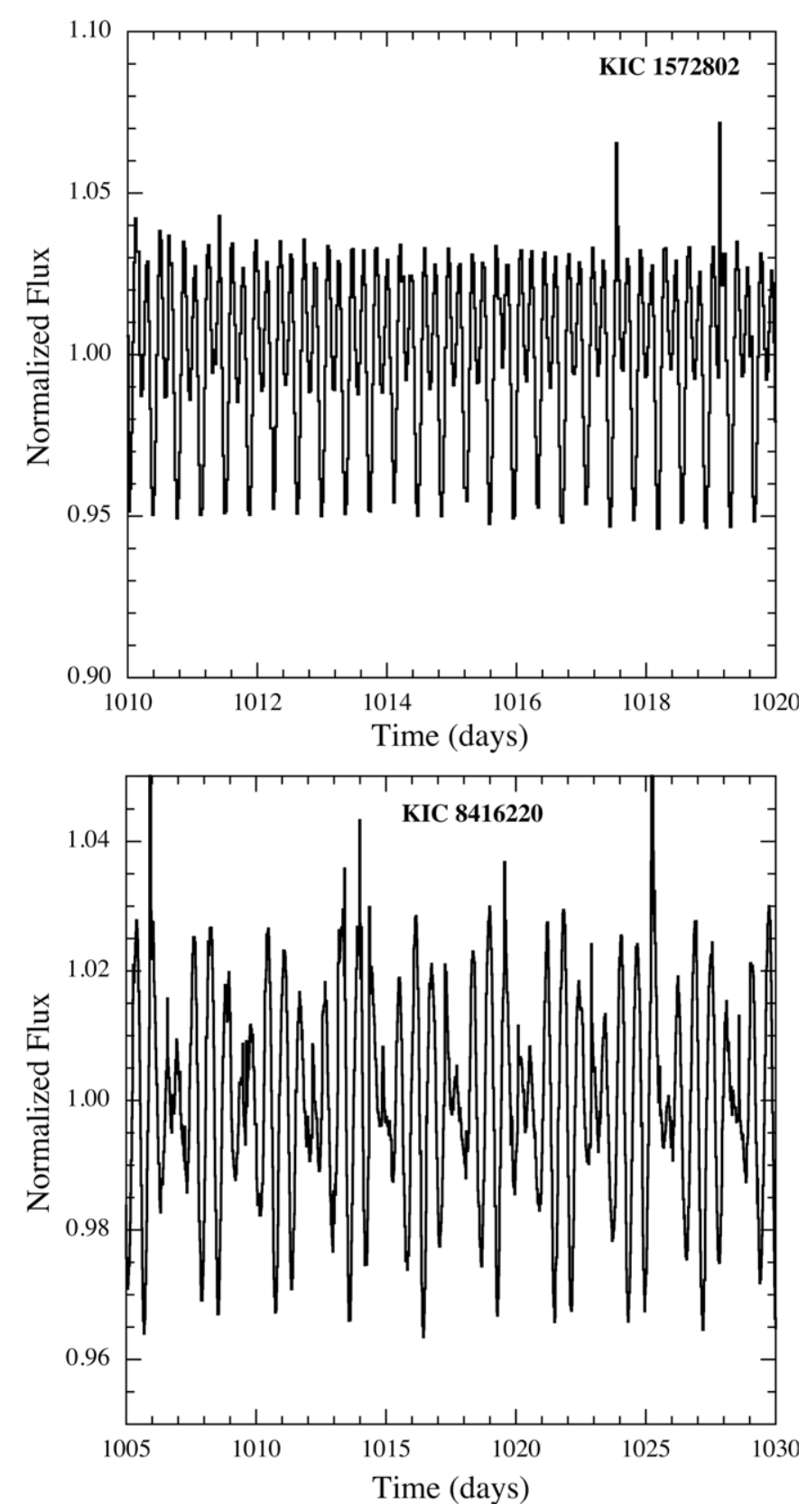

Figure 3. Short segments of the light curves for KIC 1572802 and KIC 8416220 shown in Figure 2. Top: the structure in the light curve of KIC 1572802 results from a single periodicity, but with higher harmonic structure (see Figure 4). Bottom: the structure in the light curve of KIC 8416220 results from the beat of two periodicities that differ by $\sim 23 \%$ (see Figure 4 ).

FTs below simply as "the FT." For the purpose of searching for statistically significant peaks, we further normalized the FT by dividing by a smoothed version of the FT. The smoothing was accomplished by convolution with a boxcar function that is 100 frequency bins in length. This procedure has the effect of normalizing the raw FT to its local (100-bin) mean. All targets whose normalized FT revealed at least one frequency peak exceeding the local mean by a factor $>4$ with at least one harmonic or subharmonic which exceeded its local mean by a factor of three, were considered to be worthy of further investigation. We note that the analysis outlined here of the 3897 DC13 M-star Kepler targets requires only about $1 \mathrm{hr}$ of cpu time on a standard laptop machine. 
In all, we find 297 of the 3897 targets exhibit the requisite significant FT signal comprising a base frequency plus its harmonic, with the base frequency exceeding 0.5 cycles day $^{-1}$ (i.e., $P_{\text {rot }}<2$ days). We believe that the majority of these periodicities are likely to be due to stellar rotation manifested via starspots, but a significant number may be due to planet transits and binary eclipses. The individual FTs for these systems were further examined to eliminate those which were clearly not due to rotating starspots. In all cases we folded the data modulo the detected fundamental period, and were readily able to rule out cases due to transiting planets since their well-known sharp, relatively rectangular dipping profiles are characteristic. Of course, we also checked the KOI list for matches. Any of the objects that appear in the Kepler eclipsing binary ("EB") star catalog (e.g., Matijevič et al. 2012) were likewise eliminated.

Spots on a rotating star can, depending on their locations and other properties, produce a profile that may be mistaken for the profile of an EB. The target KIC 1572802 is an example; see the top panel of Figure 3. It exhibits variations at a period of $8.9 \mathrm{hr}$ that resemble the light curve of a contact binary. However, an inspection of the top panel in Figure 2, which shows the corresponding full Kepler light curve, indicates that the object is not likely to be a contact binary. Aside from the profusion of stellar flares, which are not atypical of M stars, the overall envelope of the $8.9 \mathrm{hr}$ periodicity is seen to be highly and erratically variable. These amplitude changes are inconsistent with the behavior of a typical EB. Moreover, the shape of the $8.9 \mathrm{hr}$ modulation changes dramatically over the four years of observations. The FT for this object is shown in the top left panel of Figure 4.

The bottom panels of Figures 2 and 3 provide another example, i.e., the M star KIC 8416220 that exhibits a non-EB like light curve, yet where the overall amplitude remains nearly constant over the long term. This light curve results from a beat between two rotation periodicities whose periods differ by only $\sim 20 \%$ (see the FT in the upper right panel of Figure 4).

After carefully examining the $297 \mathrm{M}$ star targets that exhibit significant periodicities with periods less than 2 days, we believe we have identified the stellar rotators, and are able to exclude the EBs and transiting planets. In summary, we find that approximately 110 of these are previously identified binaries or transiting planets, or are likely to be binaries or transiting planets based on the characteristics of their FTs or folded light curves. Some eight of the 297 targets were identified as artifacts caused by leakage of the signal from the very bright star RR Lyr that pulsates with a period of 0.5669 day. After elimination of these targets, there remain 178 targets that exhibit one or more periodicities that are likely to be due to starspots (see Table 1) on rotating stars. These targets comprise a total of 211 different periodicities with $P_{\text {rot }} \lesssim 2$ days. Some 110 of the 178 targets exhibit 127 periodicities with $P_{\text {rot }} \lesssim 1$ day. The distribution of all 211 periods is shown in the bottom panel of Figure 1.

\section{POSSIBLE STELLAR PULSATIONS AMONG THE PERIODICITIES}

Stars with convective envelopes oscillate in multiple modes that are typically manifest as a forest of peaks in a Fourier spectrum, with amplitudes forming a roughly Gaussian-shaped envelope (see, e.g., Bedding 2014 for a recent review). The frequency of the mode with the maximum amplitude, $v_{\max }$, has been proposed to scale with the acoustic cut-off frequency (Brown et al. 1991) and can be related to basic stellar parameters by the scaling relation (Kjeldsen \& Bedding 1995):

$$
\frac{v_{\max }}{v_{\max , \odot}}=\frac{M / M_{\odot}}{\left(R / R_{\odot}\right)^{2} \sqrt{\left(T_{\text {eff }} / 5777 K\right)}} .
$$

Using the masses, effective temperatures, and radii of Kepler M-star targets as determined by Dressing \& Charbonneau (2013), as well as $v_{\max , \odot} \simeq 3140 \mu \mathrm{Hz}$ (Barban et al. 2013), this relation yields $v_{\max }$ values in the range $6.4-31.6 \mathrm{mHz}$, corresponding to periods between 0.5 and 2.6 minutes. This is at least a factor of 80 shorter than the shortest period in our stellar sample. Additionally, scaling relations for amplitudes of convectively excited oscillations predict amplitudes lower than a few ppm for $M$ dwarfs (Corsaro et al. 2013), which is incompatible with our observations. This, in combination with the lack of the typical Gaussian-shaped forests of peaks in our FTs, argues strongly against solar-like oscillations as the cause of the observed variability.

On the other hand, pulsations driven by the $\epsilon$ mechanism and convective-flux blocking similar to those in $\gamma$ Doradus variables have been theoretically predicted to occur in $\mathrm{M}$ dwarfs (Rodríguez-López et al. 2012, 2013). Usually, the associated pulsation periods would be of the order of half an hour, but for some low-mass pre-main sequence models timescales between 7 and $11 \mathrm{hr}$ were found. However, the authors argued that these oscillations would only grow during the short phase of deuterium burning, and, furthermore, would only grow slowly during that phase. It thus seems unlikely that pulsations driven by this mechanism could be responsible for the observed periodicities. Further evidence against contamination of our sample by stellar pulsations is provided in Sections 4 and 5 .

\section{TIME-FREQUENCY ("SONOGRAM") ANALYSIS}

As a further check as to whether the periodicities in the Kepler M-star data are the result of the rotation of spotted stars, we have also carried out sonogram, i.e., time-frequency, analyses. In the present case, short segments of the Kepler time series are Fourier transformed and the amplitudes in each FT are displayed as a function of frequency in a vertical linear strip of a two-dimensional image. The orthogonal coordinate of the image encodes the start times of the data segments. The duration of the individual segments of the time series, and their overlap are parameters that can be adjusted. The image shows how the amplitude of a particular signal evolves with time.

To compute the sonograms, we used the program package TiFrAn (time frequency analysis; Kolláth \& Oláh 2009) which allows a time-frequency analysis to be carried out via several different methods. From these possible options, we have chosen the short-term Fourier transform to study the rotational periodicities. This procedure yields good resolution in both time and frequency. In particular, each data segment is defined by a Gaussian window function with full width at half maximum of about 30 days, and the centers of the windows of sequential segments are separated by $\sim 2$ days.

In Figure 5 we show sonograms for the same four Kepler targets whose full FTs are displayed in Figure 4. The FT amplitudes of most of the individual frequency components appear to change dramatically and erratically over time, and largely independently of one another. This gives added weight to the hypothesis that these are indeed features due to spots on rotating stars, as opposed to stellar pulsations or other more stable periodicities, e.g., binary modulations. 
Table 1

Kepler M Stars Exhibiting a Short Rotation Period

\begin{tabular}{|c|c|c|c|c|c|c|c|}
\hline KIC & Period & KIC & Period & KIC & Period & KIC & Period \\
\hline 1572802 & 0.3711 & 6592335 & 0.4119 & 8565914 & 1.4183 & 10790812 & 1.0629 \\
\hline 2300039 & 1.7083 & 6664639 & 1.4482 & 8611876 & 1.5797 & 10790838 & 0.9173 \\
\hline 2449101 & 0.7399 & 6715960 & 0.9185 & 8672278 & 1.8549 & 10796551 & 1.1485 \\
\hline 2557669 & 1.8595 & 6752578 & 0.2601 & 8673358 & 1.0292 & 10803430 & 1.5207 \\
\hline 3130391 & 1.2301 & 6762389 & 0.3855 & 8681527 & 0.5775 & 10975238 & 1.9487 \\
\hline 3439791 & 0.4321 & 6928206 & 1.2839 & 8873575 & 0.6702 & 11031746 & 1.2109 \\
\hline 3454793 & 0.6682 & 6936046 & 0.5274 & 8909833 & 1.2812 & 11042875 & 1.5658 \\
\hline 3642335 & 0.6725 & 6949412 & 1.6453 & 8935942 & 1.6582 & 11091336 & 1.1947 \\
\hline 3732401 & 1.7990 & 7110077 & 0.9717 & 9022001 & 0.6989 & 11124203 & 0.9596 \\
\hline 3748172 & 1.0516 & 7269729 & 0.3346 & 9041966 & 0.1477 & 11140425 & 1.1879 \\
\hline 3757251 & 0.2158 & 7431659 & 1.0507 & 9075708 & 0.5131 & 11147271 & 1.8794 \\
\hline 3831911 & 0.5621 & 7434110 & 0.4014 & 9083354 & 1.1341 & 11189348 & 0.5060 \\
\hline 3935499 & 0.9214 & 7445605 & 1.0782 & 9091897 & 0.2399 & 11305240 & 0.7808 \\
\hline 3962433 & 0.4025 & 7448057 & 0.3873 & 9142641 & 1.3747 & 11343461 & 1.6167 \\
\hline 4036313 & 1.5816 & 7449695 & 0.5610 & 9142714 & 1.1285 & 11349556 & 1.6941 \\
\hline 4077867 & 0.4777 & 7547969 & 0.6802 & 9205855 & 0.9144 & 11390683 & 1.1295 \\
\hline 4246255 & 1.3177 & 7592990 & 0.4426 & 9268481 & 0.5802 & 11446073 & 1.0393 \\
\hline 4264634 & 0.7541 & 7678417 & 0.6267 & 9335198 & 0.2898 & 11447564 & 0.3812 \\
\hline 4473355 & 0.2060 & 7686474 & 1.4843 & 9395840 & 1.0973 & 11498106 & 0.5708 \\
\hline 4545041 & 0.3326 & 7733540 & 1.5488 & 9479539 & 1.8576 & 11521274 & 0.6653 \\
\hline 4660255 & 0.4109 & 7740983 & 0.4036 & 9519275 & 0.5797 & 11605209 & 1.8622 \\
\hline 4951466 & 0.1349 & 7741987 & 1.2612 & 9590249 & 0.2642 & 11668095 & 0.9156 \\
\hline 5083330 & 1.9550 & 7743830 & 0.3749 & 9702550 & 1.6270 & 11702167 & 0.3798 \\
\hline 5095098 & 0.7414 & 7800087 & 0.4722 & 9705079 & 0.5377 & 11722217 & 0.1643 \\
\hline 5182822 & 0.3391 & 7847566 & 0.3806 & 9761113 & 0.6806 & 11760021 & 0.7565 \\
\hline 5184487 & 1.1051 & 7849619 & 0.3092 & 9784820 & 1.1005 & 11855334 & 0.8173 \\
\hline 5341666 & 0.3631 & 7973675 & 0.4572 & 9899900 & 0.4145 & 11855853 & 1.4325 \\
\hline 5360129 & 1.3519 & 8012943 & 0.5726 & 9933464 & 1.2234 & 11876220 & 1.4600 \\
\hline 5435958 & 0.8370 & 8057610 & 1.7332 & 9992083 & 0.4197 & 11955208 & 0.5637 \\
\hline 5621528 & 0.7991 & 8075991 & 1.2932 & 10027247 & 0.5887 & 12022407 & 0.7976 \\
\hline 5685704 & 0.5129 & 8098178 & 0.9874 & 10324374 & 1.7311 & 12058533 & 0.3442 \\
\hline 5771150 & 0.5049 & 8098228 & 0.5472 & 10384891 & 0.4079 & 12060710 & 0.3788 \\
\hline 5937264 & 0.6037 & 8107903 & 1.5406 & 10403228 & 0.2369 & 12105694 & 1.9567 \\
\hline 5938531 & 0.8800 & 8150479 & 0.3805 & 10412044 & 1.4002 & 12105867 & 1.1736 \\
\hline 5951140 & 1.0551 & 8183594 & 0.2980 & 10462462 & 0.8696 & 12203082 & 0.5263 \\
\hline 5952378 & 0.4528 & 8248415 & 0.9892 & 10469305 & 0.8971 & 12207432 & 1.6982 \\
\hline 5962956 & 1.3998 & 8257134 & 0.2995 & 10515986 & 0.7472 & 12258225 & 0.9423 \\
\hline 6102091 & 0.5162 & 8325962 & 0.5721 & 10518758 & 1.3394 & 12304013 & 0.3498 \\
\hline 6117832 & 0.6047 & 8414250 & 0.7446 & 10536761 & 1.0255 & 12356535 & 0.8096 \\
\hline 6122790 & 1.1193 & 8415004 & 1.2297 & 10552016 & 1.5089 & 12365719 & 0.8521 \\
\hline 6183736 & 1.1819 & 8416220 & 0.5660 & 10553513 & 0.2547 & 12505054 & 0.4397 \\
\hline 6370174 & 0.8606 & 8417053 & 1.4751 & 10584063 & 1.4280 & 12784183 & 0.2087 \\
\hline 6425928 & 0.3255 & 8447096 & 1.2361 & 10587237 & 0.9603 & 12835232 & 1.8951 \\
\hline 6469920 & 0.9717 & 8454353 & 1.4905 & 10677397 & 0.3115 & & \\
\hline 6529445 & 0.2231 & 8474897 & 0.9954 & 10710753 & 0.6493 & & \\
\hline
\end{tabular}

Notes. 178 Kepler targets exhibiting at least one starspot rotation period shorter than 2 days. If more than one period is present, only the shortest period is listed here. Periods are in days. For systems with more than one short rotation period see Table 2.

\section{TRACKING THE MODULATION PHASES}

It would not be surprising to find that starspots produce modulations with phases that are quite variable, as are the amplitudes per the above discussion. Therefore, we devised a way to track the phases of the observed periodicities in order to assess their characteristics and determine whether they suggest an origin in starspots. Because the FTs of the inferred rotationally induced spot modulations have harmonics that fall off rapidly in amplitude with frequency, we model the modulations by just the base frequency and its first harmonic. We can then describe the flux locally in the time series by the expression:

$$
\mathcal{F}(t)=A+B \sin \left(\omega t+\phi_{1}\right)+C \sin \left(2 \omega t+\phi_{2}\right)
$$

where $\omega \equiv 2 \pi / P_{\text {rot }}$. For each point in the flux time series, we fit a function of the form given by Equation (2) over a short interval of time equal to 2-4 base periods of the modulations, depending on the statistical precision that is desired. For periods between one-fourth and 2 days, this corresponds to using between 25 and 400 flux points to determine 5 unknown parameters, including the phases $\phi_{1}$ and $\phi_{2}$. The phases of the base frequency and of its first harmonic are thereby computed for every point in the time series.

Results of this "phase tracking" are shown in Figure 6 for four M-star targets for which we found, in each case, a single rotation period in the range from 0.25 to 1.4 days. The phases of the base frequency are plotted in red points while those of the first harmonic are shown in blue. Portions of 

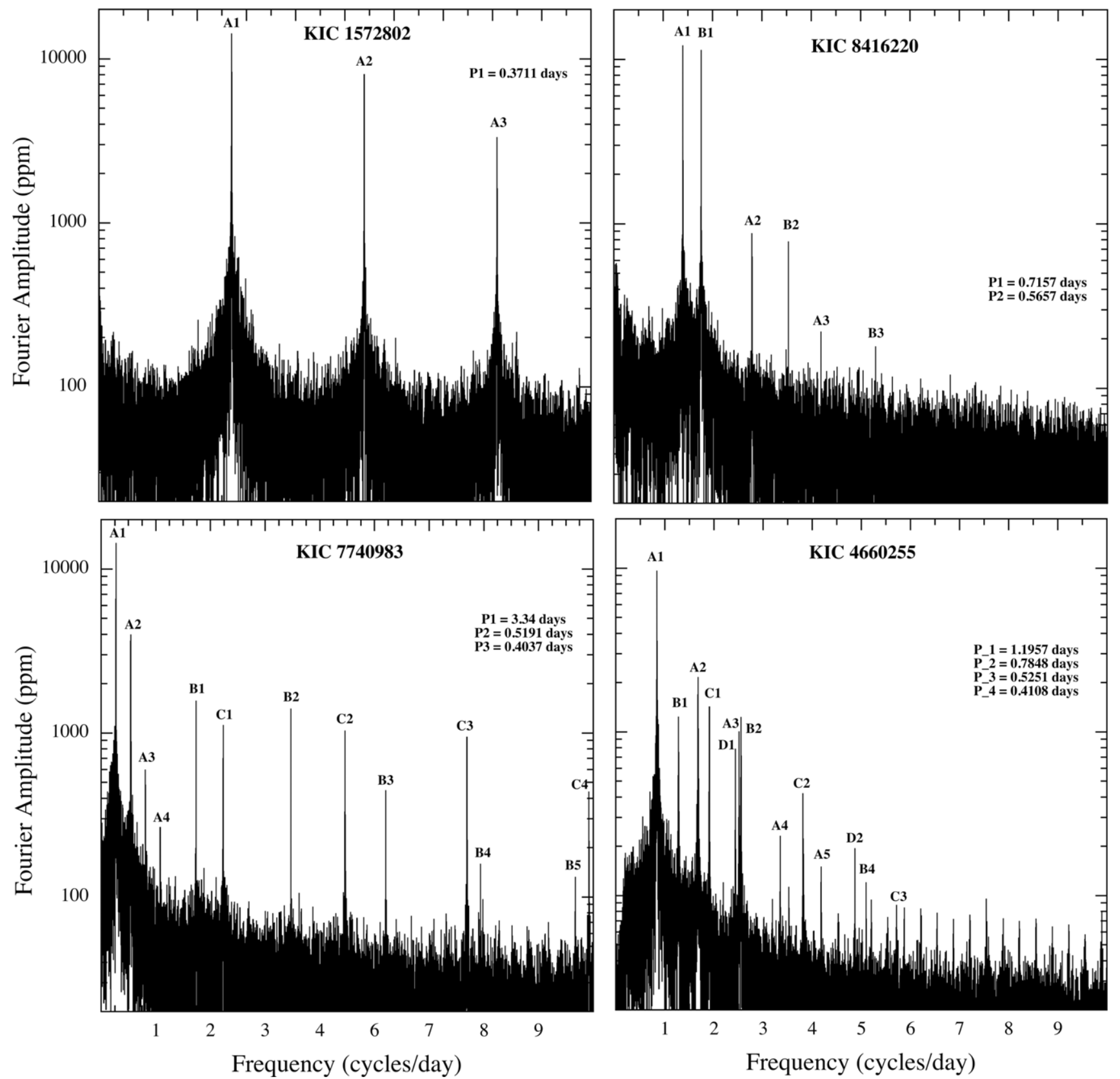

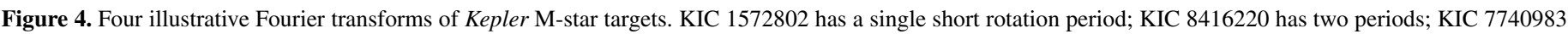

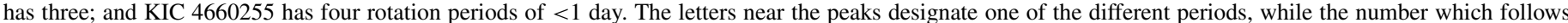

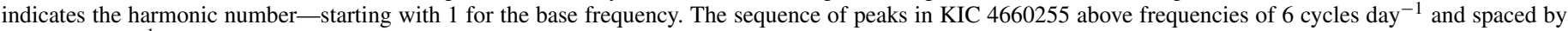
$1 / 3$ cycle day $^{-1}$ is an artifact of the Kepler momentum wheel.

the phase curves with linear trends indicate times of constant effective frequency; regions with curvature indicate changes in the apparent frequency. These plots tend to exhibit two different signatures that are likely characteristic of transitory, migrating, and differentially rotating starspots: (1) erratic phase behavior, and (2) different phase behavior for the base frequency versus the first harmonic. The stellar pulsators that we have examined with this same phase-tracking code do not behave in this manner.

More quantitatively, the phase of the modulations can be formally defined as

$$
\phi(t)=\int\left(v(t)-v_{0}\right) d t \quad \text { or } \dot{\phi}(t)=v(t)-v_{0} .
$$

In terms of the period, this can be written as

$$
P(t) \simeq P_{0}-\dot{\phi}(t) P_{0}^{2},
$$

where $v_{0}$ and $P_{0}$ are the reference frequency and period, respectively. The characteristic timescale for period changes, $\tau$, can be expressed in terms of $\ddot{\phi}$ as:

$$
\tau \equiv \frac{P}{\dot{P}} \simeq \frac{1}{P_{0} \ddot{\phi}} .
$$

As numerical examples, we note that a change in phase of 1 cycle $\left(360^{\circ}\right)$ over the Kepler mission corresponds to $\phi \simeq$ (1500 days $)^{-1}$ which implies, according to Equation (4), a difference in period from the reference period of $\sim 0.00017$ day 

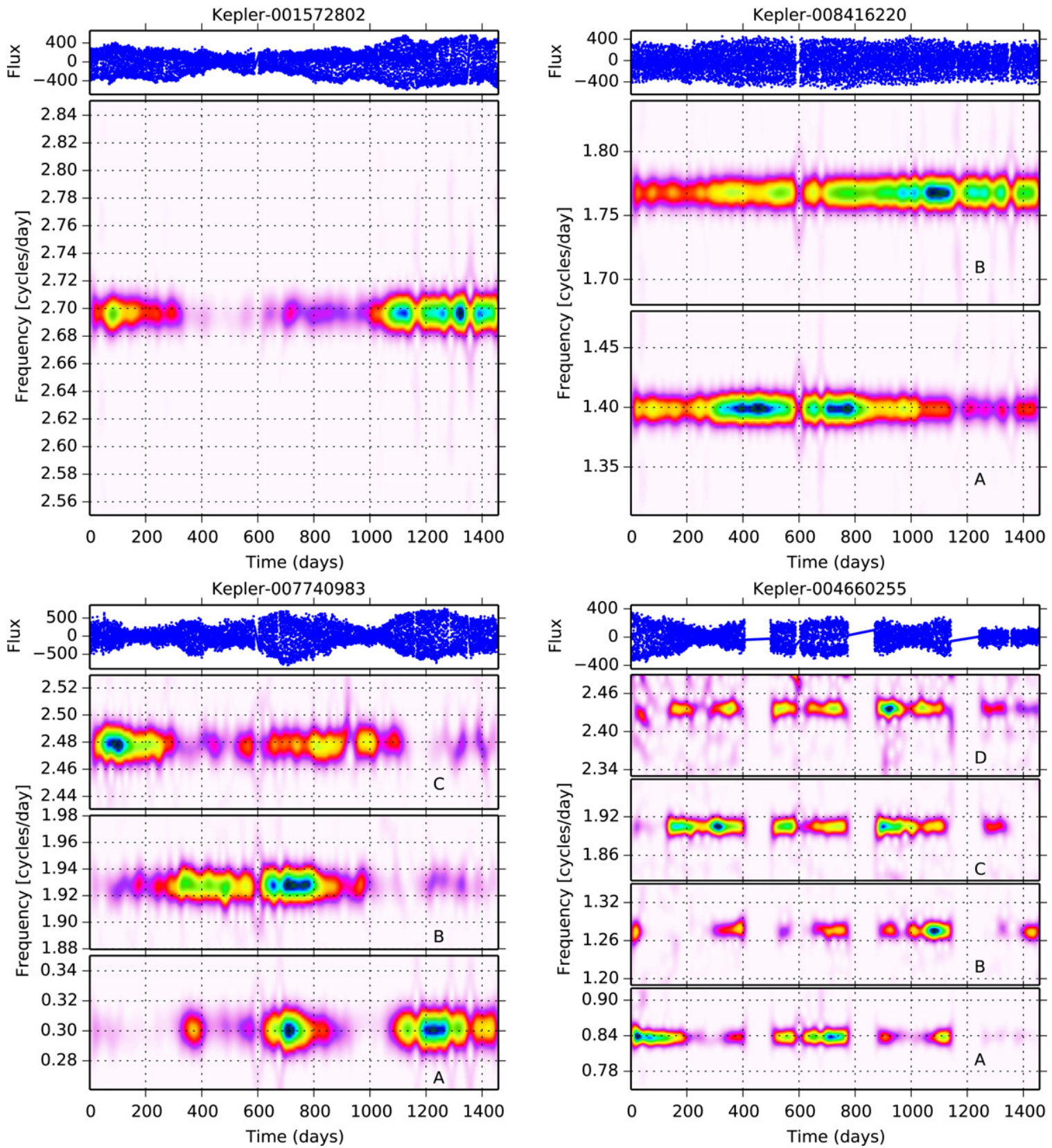

Figure 5. Illustrative "sonograms," or temporally resolved Fourier transforms, of the same Kepler M-star targets whose full FTs are shown in Figure 4. In each quadrant, the upper sub-panel shows the flux time series for the object, while the lower sub-panels show the sonogram. Each periodicity has its own intensity normalization. The color coding is related to the FT amplitude with purple/red through green/blue the lower-to-higher amplitudes, respectively. Note how most of the modulation amplitudes vary strongly with time and independently of one another. These sonograms help demonstrate that the modulations are due to rotating starspots and not binary star modulations or stellar pulsations.

(A color version of this figure is available in the online journal.)

(for $P_{0}=1 / 2$ day). In terms of the implications of curvature in the phase curves, a parabolic arc of one-sixth of a cycle in amplitude occurring over an interval of \pm 200 days corresponds to $\ddot{\phi} \simeq 8 \times 10^{-6}$ cycles day ${ }^{-2}$ with a corresponding value of $\tau \simeq 330 \mathrm{yr}$.

Finally, we note an important property of these "phase curves." By construction from Equation (2), we see that if the second harmonic term is exactly twice the base frequency, then any slopes in the phase curves should bear the relation $\dot{\phi}_{2}=2 \phi_{1}$. However we can see examples in Figure 6 where this is clearly not the case. For KIC 10553513 the two slopes are nearly the same over the final 800 days of observation, while for
KIC 7592990 the mean slope of $\phi_{2}$ is substantially larger than twice the mean slope of $\phi_{1}$. This is a direct indication that the first harmonic does not occur at exactly twice the base frequency (see also Figure 7). Vida \& Oláh (2013) and Vida et al. (2014) find that KIC 7592990 has an activity cycle on the timescale of about 500 days inferred from the systematic change of its rotational period due to differential rotation, with an estimated lower limit of $\alpha=0.0012$ (see Equation (6)). In turn, this likely demonstrates that the second harmonic may arise from spots that are located at both different longitudes and different latitudes, with the attendant differential rotation when latitude differences are present. Clustering of starspots at two active longitudes 

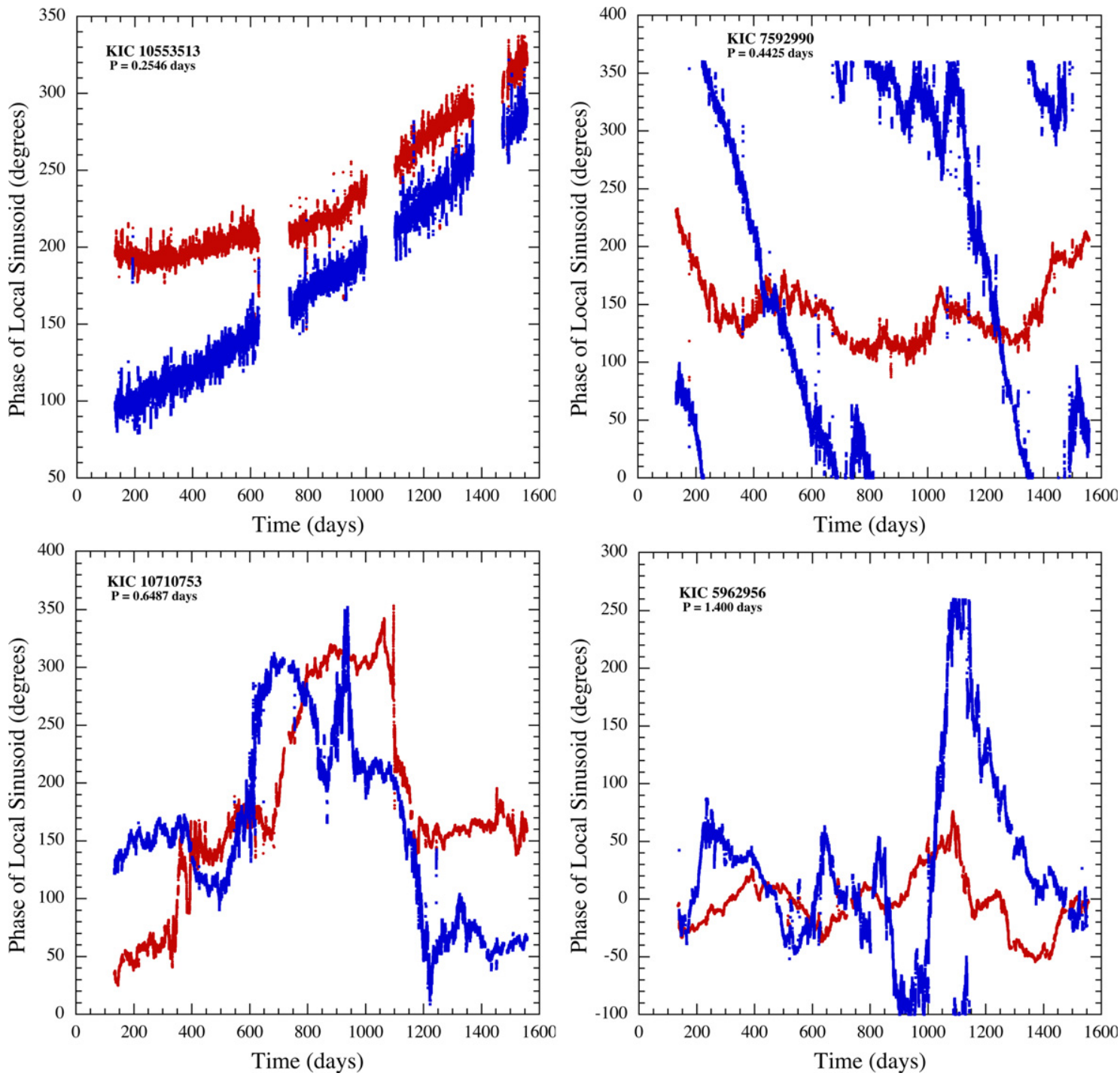

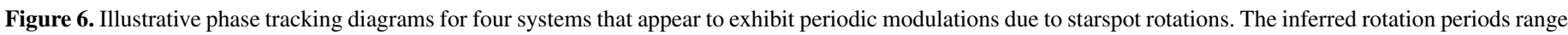

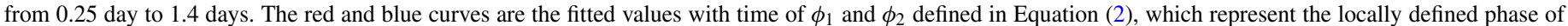

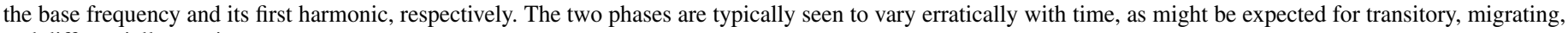
and differentially rotating starspots.

(A color version of this figure is available in the online journal.)

opposite to each other, on close binaries of dwarf stars, has been theoretically investigated by Holzwarth \& Schüssler (2003), showing that with faster rotation the occurrence of this clustering is higher (but the initial parameters of the rising flux tubes also play a significant role).

\section{MULTIPLE PERIOD SYSTEMS}

Upon further careful examination of the FTs of the 178 systems with short rotation periods among the Kepler M stars, we find $\sim 30$ objects with two or more incommensurate periods, at least 1 of which has $P_{\text {rot }}<1$ day, and a second that has $P_{\text {rot }}<2$ days. KIC 8416220 , discussed above, provides an example of an $\mathrm{M}$ star that exhibits two periodicities; see the upper right panel of Figure 4. Note that the amplitudes of all three visible harmonics at each frequency have very similar amplitudes. The two base frequencies differ by only $\sim 20 \%$.
Further scrutiny also reveals several objects with three or more independent short periods, at least one of which has $P_{\text {rot }}<$ 1 day. One particular example is KIC 7740983 whose FT is shown in the lower left panel of Figure 4. The three independent frequencies, labeled " $A$," " $B$," and " $C$ " exhibit 3,7 , and 11 harmonics, respectively, out to 25 cycles day $^{-1}$.

One of the systems whose FT we studied exhibited four independent frequencies: KIC 4660255 (see lower right panel of Figure 4). All four periods have $P_{\text {rot }}<1.2$ days.

The results of the multiple $\mathrm{M}$-star detections are summarized in Table 2 which lists 37 systems. Of these, 27 have 1 period with $P_{\text {rot }}<1$ day, another with $P_{\text {rot }}<2$ days, and each independent frequency has at least a base frequency plus the next higher harmonic. For seven of the other systems listed in Table 2, they satisfy all of these criteria, except that one of the periodicities does not have a detectable harmonic. Finally, a few remaining systems have a second period that is slightly longer than two days. 
Table 2

Kepler M Stars Exhibiting Two or More Short Rotation Periods

\begin{tabular}{|c|c|c|c|c|c|c|c|c|c|}
\hline $\begin{array}{l}\text { Object } \\
\text { (1) }\end{array}$ & $\begin{array}{c}\alpha_{\mathrm{J} 2000} \\
(2)\end{array}$ & $\begin{array}{c}\delta_{J 2000} \\
(3)\end{array}$ & $\begin{array}{l}K_{p} \\
(4)\end{array}$ & $\begin{array}{l}T_{\text {eff }} \\
(5)\end{array}$ & $\begin{array}{l}P_{A} \\
(6)\end{array}$ & $\begin{array}{l}P_{B} \\
(7)\end{array}$ & $\begin{array}{l}P_{C} \\
(8)\end{array}$ & $\begin{array}{l}P_{D} \\
(9)\end{array}$ & $\begin{array}{c}\text { Imaging } \\
(10)\end{array}$ \\
\hline 3454793 & $19^{\mathrm{h}} 36^{\mathrm{m}} 49^{\mathrm{s}} .43$ & $38^{\mathrm{d}} 31^{\mathrm{m}} 47^{\mathrm{s}} .50$ & 15.5 & 3431 & 0.6681 & 0.9673 & $\ldots$ & $\ldots$ & UKIRT: elongated (?) \\
\hline 3757251 & $19^{\mathrm{h}} 36^{\mathrm{m}} 14^{\mathrm{s}} .20$ & $38^{\mathrm{d}} 50^{\mathrm{m}} 19^{\mathrm{s}} .25$ & 15.8 & 3623 & 0.2158 & 0.4485 & 15.40 & $\ldots$ & UKIRT: $2^{\prime \prime} 6$ \& 4". 2 \\
\hline 3831911 & $19^{\mathrm{h}} 00^{\mathrm{m}} 13^{\mathrm{s}} .23$ & $38^{\mathrm{d}} 59^{\mathrm{m}} 06^{\mathrm{s}} .04$ & 15.0 & 3953 & 0.5670 & 1.8343 & $30.484^{\mathrm{c}}$ & $\ldots$ & UKIRT: 4 ". 0 \\
\hline 3962433 & $19^{\mathrm{h}} 34^{\mathrm{m}} 28^{\mathrm{s}} .26$ & $39^{\mathrm{d}} 01^{\mathrm{m}} 38^{\mathrm{s}} .78$ & 15.8 & 3741 & 0.4024 & 0.6378 & $\ldots$ & $\ldots$ & UKIRT: single \\
\hline 4077867 & $19^{\mathrm{h}} 45^{\mathrm{m}} 41^{\mathrm{s}} .35$ & $39^{\mathrm{d}} 06^{\mathrm{m}} 34^{\mathrm{s}} .56$ & 15.8 & 3490 & 0.4776 & 0.9798 & 19.35 & $\ldots$ & UKIRT: 4".0 \\
\hline 4175707 & $19^{\mathrm{h}} 42^{\mathrm{m}} 47^{\mathrm{s}} .72$ & $39^{\mathrm{d}} 16^{\mathrm{m}} 00^{\mathrm{s}} .66$ & 15.0 & 3825 & 0.3406 & 0.4163 & $\ldots$ & $\ldots$ & Keck AO: single \\
\hline 4264634 & $19^{\mathrm{h}} 28^{\mathrm{m}} 34^{\mathrm{s}} .15$ & $39^{\mathrm{d}} 18^{\mathrm{m}} 00^{\mathrm{s}} .76$ & 14.8 & 3939 & 0.7536 & 0.8865 & 12.38 & $\ldots$ & UKIRT: single \\
\hline 4545041 & $19^{\mathrm{h}} 04^{\mathrm{m}} 19^{\mathrm{s}} .82$ & $39^{\mathrm{d}} 37^{\mathrm{m}} 18.16$ & 15.9 & 3329 & 0.3321 & 0.4045 & $\ldots$ & $\ldots$ & UKIRT: single \\
\hline 4660255 & $19^{\mathrm{h}} 33^{\mathrm{m}} 17^{\mathrm{s}} .12$ & $39^{\mathrm{d}} 42^{\mathrm{m}} 32^{\mathrm{s}} .62$ & 15.4 & 3917 & 0.4108 & 0.5251 & 0.7859 & 1.1958 & UKIRT: elongated \& $4^{\prime \prime} .2$ \\
\hline 5182822 & $19^{\mathrm{h}} 22^{\mathrm{m}} 00^{\mathrm{s}} .25$ & $40^{\mathrm{d}} 22^{\mathrm{m}} 13^{\mathrm{s}} .08$ & 15.7 & 3900 & 0.3390 & 1.1660 & 15.51 & $\ldots$ & UKIRT: single \\
\hline 6425928 & $19^{\mathrm{h}} 01^{\mathrm{m}} 05^{\mathrm{s}} .40$ & $41^{\mathrm{d}} 51^{\mathrm{m}} 29.38$ & 14.5 & 3862 & 0.3254 & 0.3942 & 2.4018 & 2.9842 & UKIRT: single \\
\hline 6529445 & $19^{\mathrm{h}} 36^{\mathrm{m}} 05^{\mathrm{s}} .46$ & $41^{\mathrm{d}} 56^{\mathrm{m}} 23.42$ & 15.7 & 3746 & 0.2231 & 0.2362 & $\ldots$ & $\ldots$ & UKIRT: elongated \& 4". 7 \\
\hline 6936046 & $19^{\mathrm{h}} 10^{\mathrm{m}} 16^{\mathrm{s}} .10$ & $42^{\mathrm{d}} 26^{\mathrm{m}} 36.49$ & 15.0 & 3772 & 0.5273 & 1.2327 & 13.21 & $\ldots$ & UKIRT: $6^{\prime \prime} 6^{\mathrm{d}}$ \\
\hline 7110077 & $19^{\mathrm{h}} 17^{\mathrm{m}} 35^{\mathrm{s}} .66$ & $42^{\mathrm{d}} 41^{\mathrm{m}} 37^{\mathrm{s}} .28$ & 15.6 & 3598 & 0.9716 & 1.0390 & 22.08 & $\ldots$ & UKIRT: 2 faint compan. at $\sim 1^{\prime \prime} .3$ \\
\hline 7434110 & $19^{\mathrm{h}} 13^{\mathrm{m}} 24^{\mathrm{s}} .06$ & $43^{\mathrm{d}} 02^{\mathrm{m}} 47^{\mathrm{s}} .00$ & 16.0 & 3311 & 0.4014 & 1.8070 & $\ldots$ & $\ldots$ & UKIRT: single \\
\hline 7448057 & $19^{\mathrm{h}} 31^{\mathrm{m}} 04^{\mathrm{s}} .12$ & $43^{\mathrm{d}} 05^{\mathrm{m}} 54^{\mathrm{s}} .96$ & 15.6 & 3500 & $0.3872^{\mathrm{a}}$ & 1.9594 & $\ldots$ & $\ldots$ & UKIRT: single \\
\hline 7449695 & $19^{\mathrm{h}} 32^{\mathrm{m}} 58^{\mathrm{s}} .53$ & $43^{\mathrm{d}} 04^{\mathrm{m}} 42^{\mathrm{s}} .92$ & 16.0 & 3787 & 0.5609 & 1.6635 & $\ldots$ & $\ldots$ & UKIRT: single \\
\hline 7740983 & $19^{\mathrm{h}} 07^{\mathrm{m}} 57^{\mathrm{s}} .37$ & $43^{\mathrm{d}} 29^{\mathrm{m}} 56^{\mathrm{s}} .08$ & 14.7 & 3727 & 0.4036 & 0.5190 & 3.33 & $\ldots$ & Keck AO: $0^{\prime \prime} 32$ binary \\
\hline 7849619 & $19^{\mathrm{h}} 58^{\mathrm{m}} 02^{\mathrm{s}} .71$ & $43^{\mathrm{d}} 34^{\mathrm{m}} 02^{\mathrm{s}} .06$ & 15.5 & 3799 & 0.3092 & 0.3298 & 12.91 & $\ldots$ & UKIRT: 2 ". 6 \\
\hline 7973675 & $19^{\mathrm{h}} 45^{\mathrm{m}} 11^{\mathrm{s}} .65$ & $43^{\mathrm{d}} 45^{\mathrm{m}} 26^{\mathrm{s}} .50$ & 15.1 & 3506 & 0.4572 & 0.7764 & 17.40 & $\ldots$ & UKIRT: single \\
\hline 8325962 & $19^{\mathrm{h}} 58^{\mathrm{m}} 46^{\mathrm{s}} .21$ & $44^{\mathrm{d}} 15^{\mathrm{m}} 05^{\mathrm{s}} \cdot 62$ & 15.0 & 3946 & $0.1690^{\mathrm{a}}$ & 0.5719 & 7.721 & 12.34 & UKIRT: 6.'3 \\
\hline 8416220 & $19^{\mathrm{h}} 00^{\mathrm{m}} 57^{\mathrm{s}} .66$ & $44^{\mathrm{d}} 28^{\mathrm{m}} 27^{\mathrm{s}} .91$ & 15.1 & 3227 & 0.5659 & 0.7151 & $\ldots$ & $\ldots$ & Keck AO: 0.'27 binary \\
\hline 8701179 & $19^{\mathrm{h}} 44^{\mathrm{m}} 31^{\mathrm{s}} .15$ & $44^{\mathrm{d}} 53^{\mathrm{m}} 48^{\mathrm{s}} .08$ & 15.9 & 3900 & 0.7046 & 0.7874 & 16.18 & $\ldots$ & UKIRT: single \\
\hline 8873575 & $19^{\mathrm{h}} 07^{\mathrm{m}} 14^{\mathrm{s}} .00$ & $45^{\mathrm{d}} 07^{\mathrm{m}} 40^{\mathrm{s}} .12$ & 16.5 & 3906 & 0.6737 & 1.0226 & 11.88 & $\ldots$ & UKIRT: single \\
\hline 8909833 & $19^{\mathrm{h}} 57^{\mathrm{m}} 23^{\mathrm{s}} .98$ & $45^{\mathrm{d}} 09^{\mathrm{m}} 38.92$ & 15.6 & 3903 & 1.2802 & 7.385 & 11.80 & $\ldots$ & UKIRT: $7^{\prime \prime} \& 11^{\prime \prime d}$ \\
\hline 9022001 & $19^{\mathrm{h}} 27^{\mathrm{m}} 18^{\mathrm{s}} .51$ & $45^{\mathrm{d}} 22^{\mathrm{m}} 42^{\mathrm{s}} .02$ & 16.0 & 3648 & $0.6989^{b}$ & $0.7055^{b}$ & 14.48 & $\ldots$ & UKIRT: elongated \& 4.'0 \\
\hline 9268481 & $19^{\mathrm{h}} 02^{\mathrm{m}} 03^{\mathrm{s}} .70$ & $45^{\mathrm{d}} 44^{\mathrm{m}} 06^{\mathrm{s}} .76$ & 15.6 & 3198 & $0.5802^{\mathrm{a}}$ & 1.5780 & 1.8638 & $\ldots$ & UKIRT: single \\
\hline 9428095 & $19^{\mathrm{h}} 59^{\mathrm{m}} 37^{\mathrm{s}} .83$ & $45^{\mathrm{d}} 56^{\mathrm{m}} 04^{\mathrm{s}} .78$ & 15.9 & 3431 & 0.6289 & 0.6974 & 23.04 & $\ldots$ & UKIRT: $3{ }^{\prime \prime} 0$ \\
\hline 9519275 & $19^{\mathrm{h}} 14^{\mathrm{m}} 06^{\mathrm{s}} .86$ & $46^{\mathrm{d}} 07^{\mathrm{m}} 18^{\mathrm{s}} .77$ & 15.5 & 3578 & 0.5798 & 1.8706 & 17.71 & $\ldots$ & UKIRT: $6^{\prime \prime}$ \\
\hline 9590249 & $19^{\mathrm{h}} 30^{\mathrm{m}} 58^{\mathrm{s}} .54$ & $46^{\mathrm{d}} 13^{\mathrm{m}} 12^{\mathrm{s}} .76$ & 15.8 & 3855 & $0.2640^{\mathrm{a}}$ & 2.1665 & 12.0 & $\ldots$ & UKIRT: single \\
\hline 10403228 & $19^{\mathrm{h}} 24^{\mathrm{m}} 54^{\mathrm{s}} .41$ & $47^{\mathrm{d}} 32^{\mathrm{m}} 59^{\mathrm{s}} .93$ & 16.1 & 3386 & 0.2367 & 0.2461 & 34.6 & $\ldots$ & UKIRT: 2 ". 8 \\
\hline 10584063 & $18^{\mathrm{h}} 57^{\mathrm{m}} 06^{\mathrm{s}} .17$ & $47^{\mathrm{d}} 49^{\mathrm{m}} 28^{\mathrm{s}} .67$ & 15.7 & 3316 & $1.397^{\mathrm{a}}$ & 1.5988 & 18.72 & $\ldots$ & UKIRT: single \\
\hline 10677397 & $19^{\mathrm{h}} 45^{\mathrm{m}} 09^{\mathrm{s}} 30$ & $47^{\mathrm{d}} 54^{\mathrm{m}} 59^{\mathrm{s}} .15$ & 15.3 & 3387 & $0.3115^{\mathrm{a}}$ & 0.4743 & 7.803 & $\ldots$ & UKIRT: double \& $6^{\prime \prime} \& 7^{\prime \prime}$ \\
\hline 11955208 & $19^{\mathrm{h}} 06^{\mathrm{m}} 43^{\mathrm{s}} .50$ & $50^{\mathrm{d}} 20^{\mathrm{m}} 08^{\mathrm{s}} .77$ & 15.7 & 3604 & 0.5637 & 0.7028 & 1.1855 & $\ldots$ & Keck AO: 0.'1 binary \\
\hline 12203082 & $19^{\mathrm{h}} 12^{\mathrm{m}} 51^{\mathrm{s}} .12$ & $50^{\mathrm{d}} 48^{\mathrm{m}} 00^{\mathrm{s}} .61$ & 15.3 & 3804 & $0.5263^{\mathrm{a}}$ & 0.6592 & 11.75 & $\ldots$ & UKIRT: single \\
\hline 12258225 & $19^{\mathrm{h}} 25^{\mathrm{m}} 41^{\mathrm{s}} .94$ & $50^{\mathrm{d}} 56^{\mathrm{m}} 41^{\mathrm{s}} .82$ & 14.7 & 3500 & 0.9416 & 1.0141 & $\ldots$ & $\ldots$ & UKIRT: single \\
\hline 12304013 & $19^{\mathrm{h}} 17^{\mathrm{m}} 40^{\mathrm{s}} .27$ & $51^{\mathrm{d}} 00^{\mathrm{m}} 16.92$ & 15.2 & 3465 & 0.3497 & 2.3491 & 19.12 & $\ldots$ & UKIRT: single \\
\hline
\end{tabular}

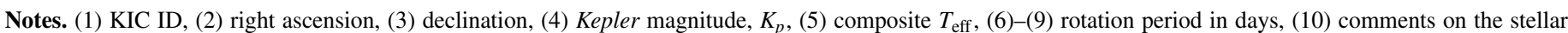
neighbors inferred from the UKIRT $J$-band images, as well as the Keck AO images-where available; angular distances to some of the neighboring stars are noted.

a Inferred from only the base frequency and no higher harmonics.

b Sufficiently close that these may result from differential rotation of a single star.

c The period of an eccentric binary component of the system.

${ }^{\mathrm{d}}$ The other stars are of a distinctly different, i.e., hotter, spectral type.

\section{BINARY AND HIERARCHICAL M-STAR SYSTEMS}

We take the presence of two or more periodicities with incommensurate periods, as described in the previous section, as evidence for the presence of two or more rapidly rotating spotted stars within the photometric aperture. This conclusion seems inevitable, since these cases are similar to the cases where only one periodicity is seen in a given target, and since we know of no evidence, for any of these periodicities, that indicates they are due to binary system modulations or stellar pulsations.

A simple statistical argument indicates that most of these targets where two or more periods have been found must consist of multiple physically related stars, i.e., stars in bound systems. Of the approximately $4000 \mathrm{M}$ stars we studied, $\sim 200$ or $\sim 5 \%$ have at least 1 rotation period shorter than 2 days, and about 100 or $\sim 2.5 \%$ have at least one rotation period shorter than 1 day. Thus, given a Kepler target that exhibits a periodicity with $P_{\text {rot }}<1$ day, the probability that a second periodicity with
$P_{\text {rot }}<2$ days will also be apparent is $\sim 5 \%$ times the chance that the image of a second M star is in the photometric aperture of the target. The probability of finding two other M stars with $P_{\text {rot }}<2$ days by chance coincidence in the aperture must be lower than $0.3 \%$. If we have examined 110 objects with $P_{\text {rot }}<1$ day, we should have found, by chance, fewer than 5 and onefourth systems with either 2 or $3 \mathrm{M}$ stars, respectively, in the same Kepler photometric aperture with $P_{\text {rot }}<2$ days. The actual values are 30 and 3 (see Table 2), both significantly in excess of what could be expected by chance. Here we have implicitly assumed that, on average, there is much less than one serendipitously occurring second or third detectable M star in any given photometric aperture.

In the above discussion and probability estimates for finding two or more rapidly rotating stars within the same Kepler photometric aperture, we made the assumption that the second and third stars were also $\mathrm{M}$ stars. If we loosen that assumption, then we can draw on the broader statistics about rotation 


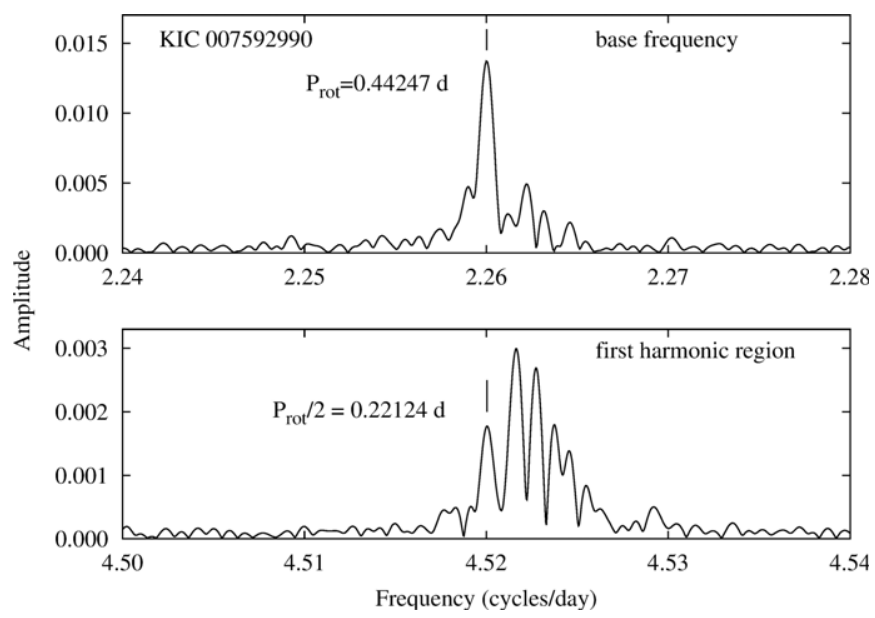

Figure 7. Zoom-in on the Fourier transform for KIC 7592990 around the base frequency and its first harmonic. Note that both peaks are broadened well beyond the frequency resolution of the 1500 day Kepler data train. The aligned vertical lines in the two panels represent the base frequency and twice the base frequency. As noted in the text, it is evident that the centroid of the first harmonic has a frequency that is clearly higher than twice that of the base frequency. This effect is also demonstrated by the phase plot in Figure 6 and indicates starspots at different latitudes undergoing differential rotation.

periods in the Kepler sample found by Reinhold et al. (2013). They identified periodic variations presumably due to spots on rotating stars, and thereby compiled the rotation periods of all active Kepler stars. Out of their sample of 21,100 stars, they found 925 with periods $\lesssim 2$ days. Thus, rapidly rotating stars comprise only $4.5 \%$ of stars across the spectral types studied by Kepler. This is essentially the same value as we found for our M-star sample, and thus the statistical argument presented above is again applicable, i.e., there is a rather low probability of finding additional rapidly rotating active stars with any of a range of spectral types in the same aperture as the first $M$ star with a short period.

We therefore adopt the working hypothesis that each $\mathrm{M}$ star target having a detected periodicity with $P_{\text {rot }}<1$ day and also one or more additional incommensurate rotational periodicities with $P_{\text {rot }}<2$, must actually consist of multiple M stars bound in a single system.

Finally, we note that 12 of the 37 systems listed in Table 2 have two short periods that are similar, i.e., they differ by no more than $4 \%-25 \%$. There is one system where two periods differ by only $1 \%$. This phenomenon can have two explanations. The first is that the two periods originate in an M-star binary with two nearly equal mass stars of essentially the same age and spindown history. Thus, perhaps the closeness of a pair of rotation frequencies is not at all unexpected.

The second possibility would be that two close periods come from spots at different latitudes of a single star that is undergoing differential rotation. Reinhold et al. (2013) have carried out an extensive study of differential rotation in 40,660 active Kepler stars. In some 18,600 of these stars they find two or more close rotation periods which they take as evidence of differential rotation and which they then use to derive limits on the differential rotation properties. Their Figure 15 summarizes the measured horizontal shear differential rotation parameter $\Delta \Omega$, which is defined as the difference in rotation frequency between the equator and the pole. For stars hotter than $\sim 6000 \mathrm{~K}$, values of $\Delta \Omega$ are often found to exceed $0.2 \mathrm{rad} \mathrm{day}^{-1}$. However, for cool stars, of the type we are studying here, $\Delta \Omega$ averages about $0.07 \mathrm{rad} \mathrm{day}^{-1}$ and only rarely exceeds $0.1 \mathrm{rad} \mathrm{day}^{-1}$. We translate this to a fractional differential rotation parameter, $\alpha$, for cool stars of arbitrary rotation rate:

$$
\alpha \equiv \frac{\Delta \Omega}{\Omega_{\mathrm{eq}}} \simeq 0.01 P(\text { days })
$$

where $\Omega_{\mathrm{eq}}$ is the equatorial rotation frequency of the star, and $P=2 \pi / \Omega_{\mathrm{eq}}$. If this relation indeed holds down to short periods (e.g., $1 / 2$ day), then it implies that any two short periods we detect which are different by more than a few percent are not likely due to differential rotation.

In any event, the possibility that some of the periodicities we see may arise on the same star should be kept in mind, though we do not expect this to be the situation in many cases. Future tests with high resolution AO imaging and ground-based spectroscopy can be of further help for checking this possibility.

Reinhold et al. (2013) also show (in their Figure 8) that there is a strong trend for stars that are of spectral-type $\mathrm{F}$ and earlier to rotate more rapidly than cooler stars which are presumably braked by magnetically constrained stellar winds (see, e.g., Mestel 1968; Skumanich 1972; Smith 1979; Zwaan 1981; Verbunt \& Zwaan 1981). Such magnetic braking likely requires dynamo activity that is stronger in stars with convective envelopes. Therefore, according to Barnes \& Sofia (1996) and Barnes (2007), M stars that are rotating with periods shorter than 2 days are probably relatively young, i.e., $\lesssim$ one to a few hundred Myr (see also Section 10).

An alternative to the "youth hypothesis" is that the short periods we see are indeed due to spots on rotating stars, but in tidally locked close binaries. One such example is V405 And with active components consisting of M0V+M5V spectral types and rotational/orbital periods of 0.465 day (Vida et al. 2009). Since the light curves of our short period systems do not exhibit obvious eclipses or ellipsoidal light variations, ${ }^{12}$ this would imply that we are viewing the systems at small orbital inclination angles. However, that would make the situation even more extreme in the sense that each such short period would itself require a binary. Thus, in systems where we see three or four short periods, this hypothesis would require six to eight bound stars, which is rather implausible.

\section{IMAGING EVIDENCE FOR MULTIPLICITY}

\subsection{UKIRT Images}

We have inspected the UKIRT $J$-band images ${ }^{13}$ for the 37 systems listed in Table 2 which exhibit 2 to 4 rotation periods. In general, for the UKIRT images of the Kepler field, one can easily distinguish two stars of comparable brightness that are separated by $\gtrsim 1 . .5$, while for objects separated by less than $\approx 1^{\prime \prime}$, there is a single image. Often, it is possible to discern that the image is elongated for stellar separations as small as $\approx 0$.'5. Of the UKIRT $J$-band frames, 19 appear to show single stellar images. However, 11 show an apparent companion, or more than 1 companion, with a separation of $\lesssim 5^{\prime \prime}$, while seven of these have separations $\lesssim 3^{\prime \prime}$. Thus, there is tentative evidence for multiplicity in the UKIRT images of $\approx 30 \%$ of the systems in Table 2.

\footnotetext{
12 In ellipsoidal variations the main peak in the FT occurs at twice the orbital frequency, $2 v$, with very small contributions at $v$, and $3 v$. We see no such cases. There is also the possibility that the amplitudes at $v$ and $3 v$ are too low to be detected, leaving only the peak at $2 v$. Several cases of frequencies with no other harmonics are indeed seen, but these are clearly marked in Table 2.

$13 \mathrm{http}: / /$ keplerscience.arc.nasa.gov/ToolsUKIRT.shtml.
} 

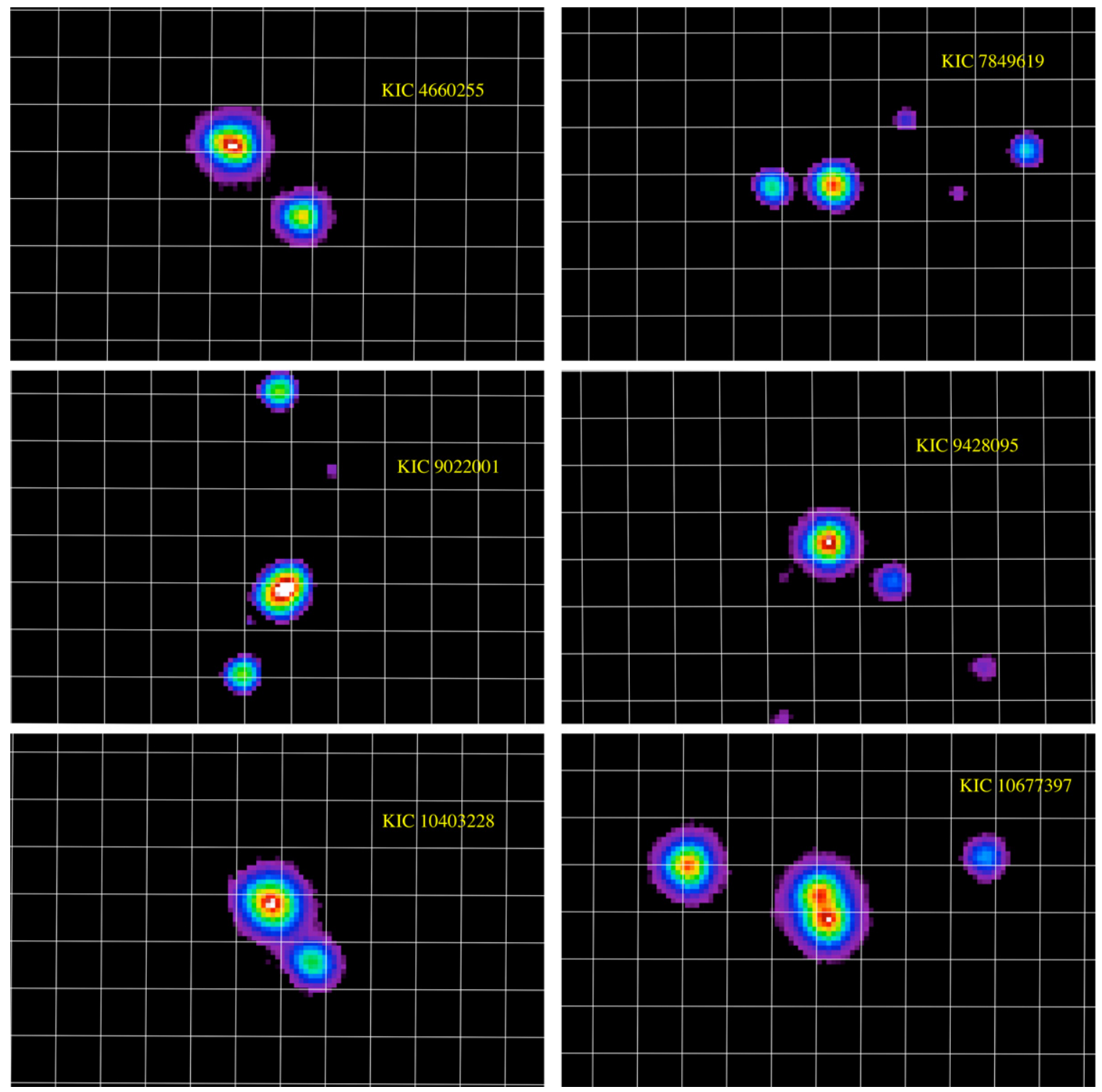

Figure 8. Selected set of UKIRT J-band images of Kepler M-stars which exhibit multiple rotation periods. From left to right and then down, they are KIC 4660255 , KIC 7849619, KIC 9022001, KIC 9428095, KIC 10403228, and KIC 10677397. The white grid lines are separated by $2^{\prime \prime} \times 2^{\prime \prime}$. In all cases, north is up and east is to the left. The images have been slightly smoothed with a two-dimensional Gaussian with $\sigma=0$ '.2. The color scale is related to the square root of the flux. KIC 7849619, KIC 9428095, and KIC 10403228 appear as fairly close doubles, i.e., with separations of $\sim 2$ 2. 5-3.'0. KIC 10677397 has a barely resolved stellar core (separation $\sim 1^{\prime \prime}$ ) and has two possible stellar companions at $6^{\prime \prime}$ and $7^{\prime \prime}$. KIC 9022001 and KIC 4660255 are apparent doubles separated by $\sim 4^{\prime \prime}$, and, in each case, the brighter star is elongated and may itself be a binary.

(A color version of this figure is available in the online journal.)

In reviewing the evidence, it is good to keep in mind that a separation of $1^{\prime \prime}$ at typical distances of $\sim 200$ pc corresponds to a physical separation of 200 AU. If, for sake of argument, bound stellar pairs are taken to have orbital separations between $0.01 \mathrm{AU}$ and $10^{5} \mathrm{AU}$ that are distributed uniformly in terms of the logarithms of the separations (e.g., Dhital et al. 2010), then fewer than half of them will have orbital separations $\gtrsim 200 \mathrm{AU}$. Thus, the UKIRT image multiplicity numbers noted above do not rule out the possibility that all of the 37 multiple M-star candidates are in bound hierarchical systems (see, e.g., Section 8.2 for some examples of closer pairs).

In Figure 8 we present six examples of multiple M-star candidates that show direct evidence for multiple stellar images within the Kepler photometric aperture. These systems are: KIC 4660255, 7849619, 9022001, 9428095, 10403228, and 10677397, and they are included in Table 2 along with their periods. The Kepler magnitudes of these stars range from 15.3 to 16.1. The magnitudes and densities of stellar images in Figure 8 indicate that most of these pairs of images are likely to be physically related as opposed to chance alignments. In Figure 9 we show the probability of interlopers (i.e., background or foreground stars) coming within a given angular distance of any particular Kepler target star. These estimates were obtained by calculating a uniform stellar density from the 10th release of the KIC $(\mathrm{KIC} 10)^{14}$ for a given magnitude limit within a 115 square degree field of view (Borucki et al. 2010). The mean stellar densities are likely slight overestimates since the KIC10 catalog includes roughly 36,000 calibration stars outside the Kepler field of view. Although the stellar density is not uniform over Kepler's field of view-rather it varies with

\footnotetext{
$\overline{14}$ http://archive.stsci.edu/kepler/kic10/search.php
} 


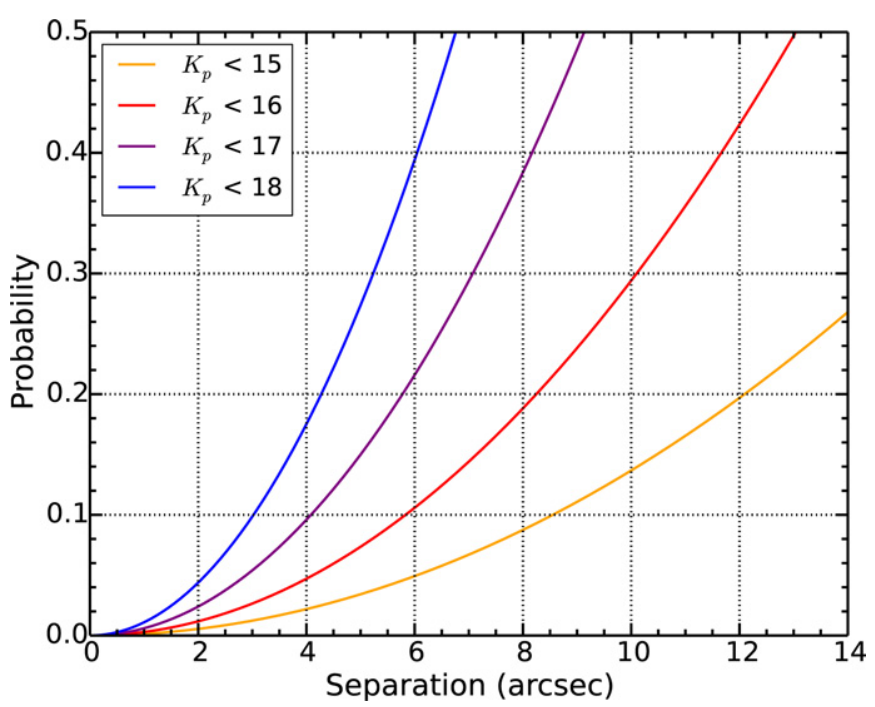

Figure 9. Probability of chance alignment of background or foreground stars with our Kepler M-star target, brighter than the indicated magnitude, as a function of separation.

(A color version of this figure is available in the online journal.)

Galactic latitude - the distribution of our multi-period sources are uniform in Galactic latitude supporting our conclusion that the multi-period sources are most likely bound companions rather than interlopers. We can see from Figure 9 that for stars comparable to, or up to 1-2 mag fainter than, our $K_{p}=$ 15-16 mag target $M$ stars, it is worth considering stars out to $\sim 5^{\prime \prime}$ as possibly physically related to the $\mathrm{M}$ star in question.

The white grid lines in Figure 8 are drawn with a spacing of $2^{\prime \prime} \times 2^{\prime \prime}$. The images have been slightly smoothed with a two-dimensional Gaussian with $\sigma=0$ '! 2 . KIC 7849619, KIC 9428095, and KIC 10403228 appear as fairly close doubles with separations of $\sim 2$.'5-3."0. KIC 10677397 has a barely resolved stellar core (separation $\sim 1^{\prime \prime}$ ) and has two possible stellar companions at $6^{\prime \prime}$ and $7^{\prime \prime}$. KIC 9022001 and KIC 4660255 are apparent doubles separated by $\sim 4^{\prime \prime}$, and, in each case, the brighter star is elongated and may itself be a binary. Finally, we remind the reader that KIC 4660255 is the remarkable object that exhibits four independent periods, all $<1.2$ days. This target is discussed in detail in Section 9.

Some of these separations seen in the UKIRT images are sufficiently large, i.e., $2^{\prime \prime}-5^{\prime \prime}$, that the "PSF" fitting technique (M. Still \& T. Barclay 2014, in preparation) may be useful for identifying which of the stellar images is the source of a particular periodicity. This method is applied to KIC 4660255 in Section 9.

\subsection{Keck AO Images}

Images of four targets in our sample were obtained with the NIRC2 camera using the Keck II AO system during the past ground-based observing season for the Kepler field. We observed KIC 4175707, KIC 11955208, and KIC 8416220 on 2013 May 30 (UT) using the laser guide star to close the AO system control loops (Wizinowich et al. 2006). The targets were imaged in $K^{\prime}$ at low air mass ( $\left.\sec z<1.1\right)$ using a threepoint dither pattern with $2^{\prime \prime}$ dither spacings. Three images were obtained at each dither position with integration times of 2, 3, and $2 \mathrm{~s}$ incorporating 10,5 , and 10 coadds per frame for a total integration time per target of 180, 135, and $180 \mathrm{~s}$, respectively. KIC 7740983 was imaged on the night of 2013 August 18 with Keck II/NIRC2 natural guide star AO (Wizinowich et al. 2000) in the $K_{s}$ band. The same three-point dither was performed for this target with $3 \mathrm{~s}$ integrations, and 10 coadds per frame for a total integration time of $180 \mathrm{~s}$.

Each dither frame was corrected for bad pixels, sky subtracted, and flat fielded. Centroids were obtained by fitting the core of the Airy pattern with a two-dimensional Gaussian function. The final images in Figure 10 show the medians of the aligned dither stacks for each target.

Each of these four targets shows a pair of rotation periods (in days) that are shorter than 1 day, viz., $\{0.57,0.72\},\{0.40,0.52\}$, $\{0.56,0.70\}$, and $\{0.34,0.42\}$, for KIC 8416220, KIC 7740983, KIC 11955208, and KIC 4175707, respectively. Amazingly, three of the four systems show close twin pairs of $M$ stars in what are almost certainly bound binaries if not higher-order multiples. Only KIC 4175707 still appears single at the $0{ }^{\prime} 05$ level.

\section{SPECIAL CASE OF KIC 4660255}

In this section we examine KIC 4660255, which exhibits four rotational periodicities all with periods shorter than 1.2 days. As the upper left panel of Figure 8 shows, at the location of KIC 4660255 there are two point-like images that are separated by 4 .'2, and, hence, are well within the photometric aperture. M. Still \& T. Barclay (2014, in preparation) have devised a "PSF" fitting technique that can utilize data at the pixel level to compute light curves for each of several stars within the photometric aperture. In brief, we assume here that the two stars can be characterized as two PSFs with two unknown fluxes and shifts of the photometric aperture relative to the sky, $\Delta \alpha$ and $\Delta \delta$ (see Figure 11). We then minimize upon the uncertaintyweighted residuals between the pixel-level data and the fit in order to yield the flux and pixel position of both stars for every one of 50,575 exposures across quarters Q1-16. This technique was demonstrated in the case of KOI 2700 to determine which of two stars within the photometric aperture was the source of planet transits (Rappaport et al. 2014).

We have carried out a PSF analysis for KIC 4660255 to try to identify from which of the two well-separated images (see Figure 8) the various rotation frequencies arise. We show in Figure 11 an example of a single frame of pixel-level data for this object. The upper left panel shows the $4^{\prime \prime} \times 4^{\prime \prime}$ Kepler pixels within the pixel mask for KIC 4660255 for a single frame of the 50,575 available for this object. The best fitting PSF model, comprised of two stars, is shown in the upper right panel. When this model is integrated over the pixels, the "fit" is shown in the lower left panel. The residuals with respect to the data from that frame, are shown in the lower right panel. This process was repeated for each of the 50,575 frames for KIC 4660255, thereby yielding two photometric time series, one for each star.

The resultant FTs for the time series extracted from the two stars within the aperture of KIC 4660255 are shown in Figure 12. The top panel shows the FT for the brighter of the two stars (the more northerly one). Only the $A$ and $B$ frequencies are present at a detectable level. The corresponding periods are 1.1958 days and 0.7859 day. No evidence is seen for the shorter $C$ and $D$ periods. In contrast, the bottom panel in Figure 12 shows the $C$ and $D$ periods, 0.5251 and 0.4108 day, very clearly. In addition, the first two harmonics of the $A$ periodicity leak through weakly - at about one-fifth of the amplitude that they exhibit in the upper panel. Thus, the PSF fitting technique appears to have cleanly distinguished the four periodicities as being from two different stellar sources. In turn, we can marginally discern from Figure 8 that the northerly image which 


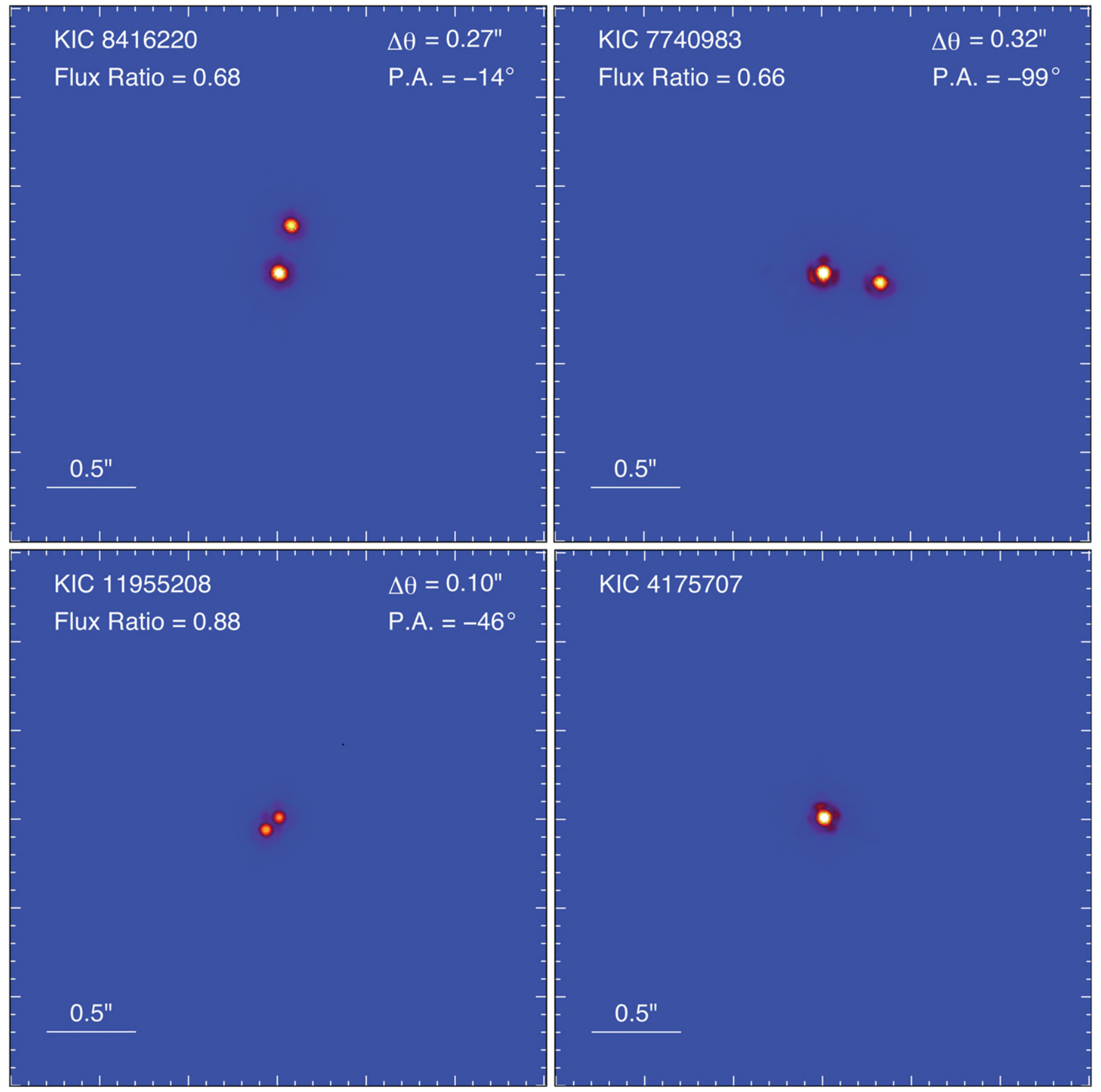

Figure 10. Keck adaptive optics images for four of our candidate multiple M-star systems. North is up and east is to the left. KIC $8416220,11955208,4175707$ were recorded at $K^{\prime}$ band, while the image of 7740983 was taken in the $K_{s}$ band. All except KIC 4175707 are obvious close twin binary M stars. The angular separations between components ranges between $0^{\prime \prime} 1$ and $00^{\prime} 32$, corresponding to physical separations of $\sim 20-65 \mathrm{AU}$ at nominal distances of 200 pc. Flux ratios and position angles of the two components in degrees east of north are displayed where applicable.

(A color version of this figure is available in the online journal.)

is host to periods $A$ and $B$ likely comprises two images, and we propose that each of those is the source of one of the $A$ and $B$ periods. By analogy, we hypothesize that the fainter, more southerly image is itself a binary, each star of which is rotating with the $C$ or $D$ period. From this analysis we conclude that, in fact, KIC 4660225 is a bound quadruple system.

\section{SUMMARY AND CONCLUSIONS}

In this work we have searched the Kepler photometric data base of M stars for short rotation periods. We found 178 Kepler targets with 211 different periods of $P_{\mathrm{rot}}<2$ days and 110 systems with 127 different periods with $P_{\text {rot }}<1$ day. Some 30 of these objects exhibit 2 or more rotation periods where at least 1 has $P_{\text {rot }}<1$ day and the other has $P_{\text {rot }}<2$ days. We also find several systems with three or more rotation periods where at least one has $P_{\text {rot }}<1$ day and the others have $P_{\text {rot }}<2$ days. There are a sufficient number of these with more than one short rotation period to allow us to argue that they are likely young, physically related, binary or hierarchical triple systems.

At least 6, and perhaps as many as 14, of these M-star systems show, in the UKIRT Kepler region survey $J$-band 


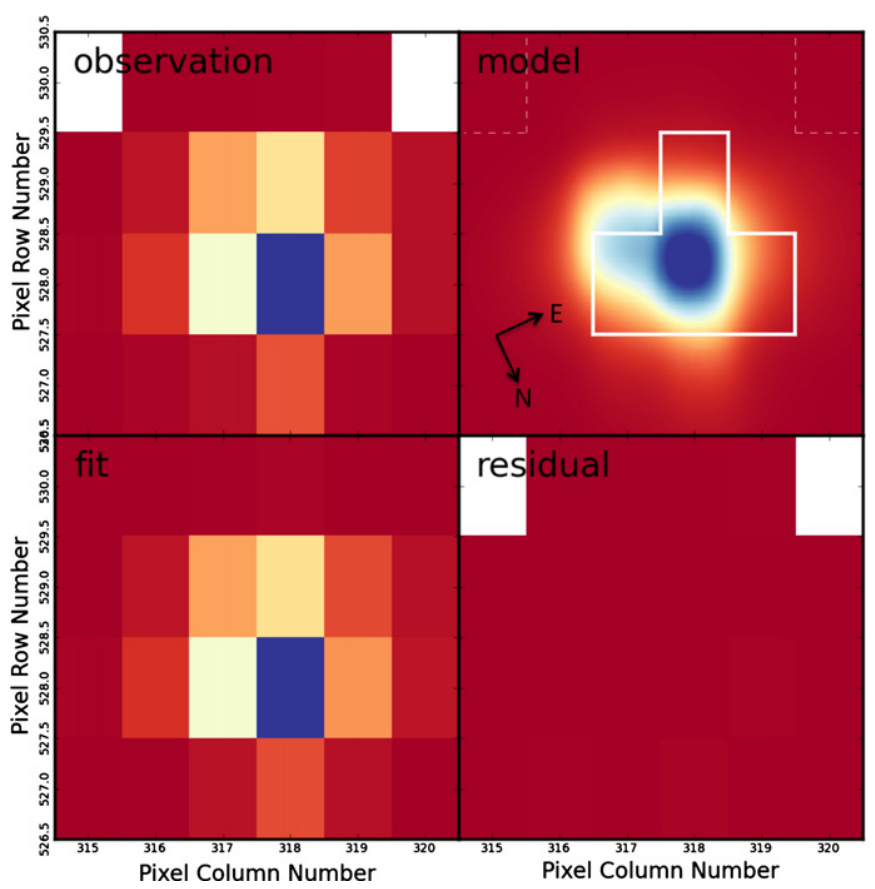

Figure 11. Typical 29.4 minute long-cadence observation of KIC 4660255, at the pixel level (each pixel is $4^{\prime \prime} \times 4^{\prime \prime}$ ), for one particular frame of $\sim 50,000$ exposures. The pixel mask for KIC 4660255 contains at least two stars- the bright blue pixel at $\{318,528\}$ as well as a fainter companion star that is about $4^{\prime \prime}$ to the left (see Figure 8). The best PSF model fit is plotted in the top right, while that fit, rebinned across the detector pixels, is compared at the lowerleft. The lower right panel contains the residuals between the data and the best fit. All images are plotted on a linear intensity scale. PSF-derived light curves are constructed by repeating this fit for every exposure over quarters $1-16$. The objective is to disentangle the fluxes between the two stars within the pixel mask. The approximate orientation of the pixels with respect to the sky is indicated by the compass in the upper right panel. The inverted " $T$ "-shaped region in the same panel indicates a typical pixel-level photometric aperture for this target used for conventional analyses.

(A color version of this figure is available in the online journal.)

images, multiple stellar images ${ }^{15}$ that may represent physically related stars. Three more systems were clearly resolved in Keck AO images of four systems. These are almost certainly physically bound twin M-star binaries. It seems quite likely that additional AO imaging in the future will reveal that more of these 30 multiple-period systems contain multiple stellar images. Spectroscopy could further reveal closer binary members.

If most of these M-star systems with multiple short periods indeed turn out to be multiple bound M-stars, this could prove a valuable way of discovering young hierarchical M-star systems. We suggest that this approach may also be applicable to $\mathrm{K}$ and G stars.

In the process of conducting this study we found that approximately $5 \%$ of all $\mathrm{M}$ stars are rotating with periods shorter than two days. If we utilize standard models of contraction onto the main sequence, with the consequent spinup of the star due to conservation of angular momentum, and the loss of systemic angular momentum due to magnetic braking (see, e.g., Kawaler 1988; Chaboyer et al. 1995; Barnes \& Sofia 1996; Irwin et al. 2011) we can check whether $M$ stars spend sufficient time rotating rapidly to allow for $5 \%$ of them to have $P_{\text {rot }} \lesssim 2$ days. These models typically take the magnetic braking torque to be proportional to $\omega_{\text {rot }}^{3}$ when $\omega_{\text {rot }}<\omega_{\text {sat }}$, where $\omega_{\text {sat }}$ is a "saturation"

15 These 14 systems are ones with another stellar image within $\sim 5^{\prime \prime}$ of the target and within $\sim|\Delta J| \lesssim 2$.
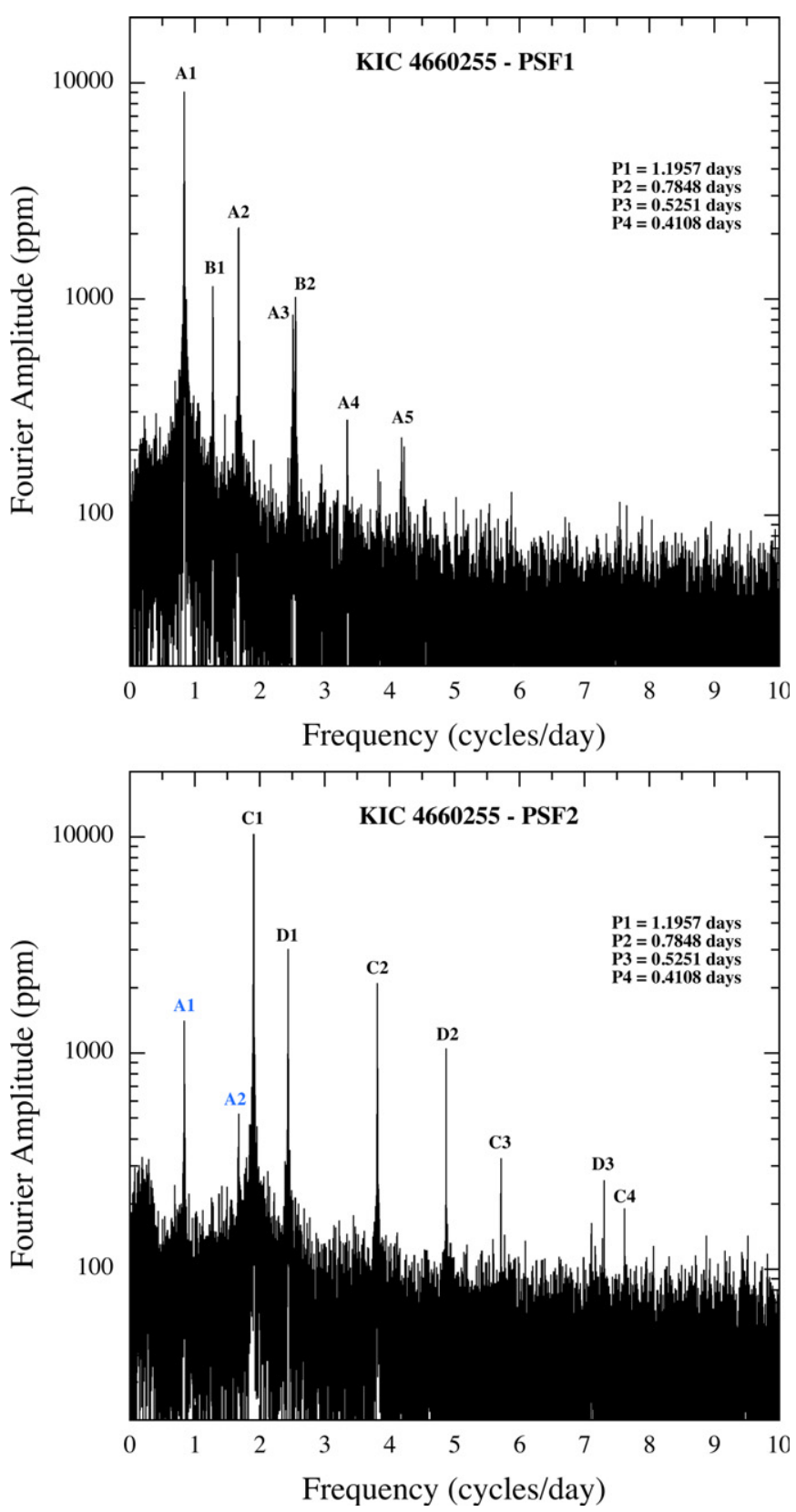

Figure 12. Fourier transforms of the PSF analyzed data trains for KIC 4660255. Top: this is the PSF-extracted data train for the brighter of the two stars within the pixel mask. Note that of the four periodicities in this Kepler target, only the longer two periods show up clearly. Bottom: PSF-extracted data train for the fainter of the two stars within the pixel mask. Here, the shorter two periods show up prominently, with only a small "leakage" of the brighter star and its period of 1.19 days. Thus, we associate two rotation periods with the brighter star and the other two with the fainter star.

(A color version of this figure is available in the online journal.)

frequency, above which the torque is proportional to $\omega_{\text {rot }} \omega_{\text {sat }}^{2}$. As an example of a model spin history of an M star, see Figure 13 of Irwin et al. (2011; dashed curve). We see that a minimum rotation period of 0.3 day is attained at $\sim 100 \mathrm{Myr}$, and the period lengthens to more than 1 day at $300 \mathrm{Myr}$ and 2 days at 400 Myr. Thus, at least for this specific example, which involves numerous uncertainties, the fraction of time that a $10^{10} \mathrm{yr}$ old M star has spent with $P_{\text {rot }} \lesssim 2$ days is about $4 \%$ of its lifetime. This is reassuringly consistent with the fraction of $\mathrm{M}$ stars that we find are rotating rapidly. 
We acknowledge several useful discussions about the particular M star(s) KIC 7740983 with Robert Szabo and Katrien Kolenberg. We also thank Cristina Rodriguez-Lopez, Jim MacDonald, Jérôme Quintin, Alex Brown, Günter Houdek, and Lucianne Walkowicz for important discussions about the possibility of observing M-star pulsations with Kepler. Arthur Delarue wrote a very helpful code for automatically extracting significant incommensurate frequencies from FTs. We thank Sasha Hinkley and Benjamin Montet for performing a subset of our Keck AO observations. We are grateful to the Kepler team for providing such valuable data to the community. D.H. acknowledges NASA support through the Kepler Participating Scientist Program under grant NNX14AB92G. R.S.O. acknowledges support through the Kepler Participating Scientist Program and the NASA Origins Program under grants NNX12AC76G and NNX11AG85G. P.S.M. acknowledges support from the Hubble Fellowship Program, provided by NASA through Hubble Fellowship grant HST-HF-51326.01-A awarded by the STScI, which is operated by the AURA, Inc., for NASA, under contract NAS 5-26555. G.H. is grateful for support by the Polish NCN grant 2011/01/B/ST9/05448. K.O. and K.V. acknowledge support from the Hungarian OTKA grants K-81421 and K-109276, and from "Lendület-2012" Young Researchers' Programs of the Hungarian Academy of Sciences. This research has made use of the NASA Exoplanet Archive, and the Mikulski Archive for Space Telescopes (MAST). We made use of $J$-band images that were obtained with the United Kingdom Infrared Telescope (UKIRT) which is operated by the Joint Astronomy Centre on behalf of the Science and Technology Facilities Council of the U.K. Some of the data presented herein were obtained at the W. M. Keck Observatory, which is operated as a scientific partnership among the California Institute of Technology, the University of California and the National Aeronautics and Space Administration. The Observatory was made possible by the generous financial support of the W. M. Keck Foundation. We acknowledge the very significant cultural role and reverence that the summit of Mauna Kea has always had within the indigenous Hawaiian community, and we are most fortunate to have the opportunity to conduct observations from this mountain.

\section{REFERENCES}

Baraffe, I., Chabrier, G., Allard, F., \& Hauschildt, P. H. 2002, A\&A, 382, 563 Barban, C., Beuret, M., Baudin, F., Goupil, M.-J., \& Samadi, R. 2013, JPhCS, 440, 012031

Barnes, J. R., Collier Cameron, A., Donati, J.-F., et al. 2005, MNRAS, 357, L1 Barnes, S., \& Sofia, S. 1996, ApJ, 462, 746

Barnes, S. A. 2007, ApJ, 669, 1167
Batalha, N. M., Borucki, W. J., Koch, D. G., et al. 2010, ApJ, 713, 109 Batalha, N. M., Rowe, J. F., Bryson, S. T., et al. 2013, ApJS, 204, 24 Bedding, T. 2014, Asteroseismology; Part of the Canary Islands Winter School of Astrophysics ed. L. Pallé \& C. Esteban (arXiv:1107.1723)

Birkby, J., Nefs, B., Hodgkin, S., et al. 2012, MNRAS., 426, 1507

Borucki, W. J., Koch, D., Basri, G., et al. 2010, Sci, 327, 977

Borucki, W. J., Koch, D. G., \& Basri, G. 2011, ApJ, 736, 19

Boyajian, T. S., von Braun, K., van Belle, G., et al. 2012, ApJ, 757, 112

Brown, T. M., Gilliland, R. L., Noyes, R. W., et al. 1991, ApJ, 368, 599

Brown, T. M., Latham, D. W., Everett, M. E., \& Esquerdo, G. A. 2011, AJ, 142,112

Burke, C. J., Bryson, S. T., Mullally, F., et al. 2014, ApJS, 210, 19

Chaboyer, B., Demarque, P., \& Pinsonneault, M. H. 1995, ApJ, 441, 865

Chabrier, G. 2003, PASP, 115, 763

Corsaro, E., Fröhlich, H. E., Bonnano, A., et al. 2013, MNRAS, 430, 2313

Cruz, K. L., \& Reid, I. N. 2002, AJ, 123, 2828

Dhital, S., West, A. A., Stassun, K. G., \& Bochanski, J. J. 2010, AJ, 139,2566

Dressing, C. D., \& Charbonneau, D. 2013, ApJ, 767, 95

Henry, T. J., Jao, W.-C., Subasavage, J. P., et al. 2006, ApJ, 132, 2360

Henry, T. J., Kirkpatrick, J. D., \& Simons, D. A. 1994, AJ, 108, 1437

Holzwarth, V., \& Schüssler, M. 2003, A\&A, 405, 303

Irwin, J., Berta, Z. K., Burke, C. J., et al. 2011, ApJ, 727, 56

Kawaler, S. D. 1988, ApJ, 333, 236

Kjeldsen, H., \& Bedding, T. R. 1995, A\&A, 293, 87

Koch, D. G., Borucki, W., Basri, G., et al. 2010, ApJL, 713, L79

Kolláth, Z., \& Oláh, K. 2009, A\&A, 501, 695

Küker, M., \& Rüdiger, G. 2011, AN, 332, 933

Mann, A. W., Gaidos, E., Kraus, A., \& Hilton, E. J. 2013, ApJ, 770, 43

Mann, A. W., Gaidos, E., Lépine, S., \& Hilton, E. J. 2012, ApJ, 753, 90

Matijevič, G., Prša, A., Orosz, J. A., et al. 2012, AJ, 143, 123

McQuillan, A., Aigrain, S., \& Mazeh, T. 2013, MNRAS, 432, 1203

Mestel, L. 1968, MNRAS, 138, 359

Morton, T. D., \& Swift, J. J. 2013, arXiv:1303.3013

Muirhead, P. S., Hamren, K., Schlawin, E., et al. 2012, ApJL, 750, L37

Rappaport, S., Barclay, T., DeVore, J., et al. 2014, ApJ, 784, 40

Reid, R. I., Gizis, J. E., \& Hawley, S. L. 2002, AJ, 124, 2721

Reiners, A. 2006, A\&A, 446, 267

Reinhold, T., Reiners, A., \& Basri, G. 2013, A\&A, 560, 4

Rodríguez-López, C., MacDonald, J., Amado, P. J., Moya, A., \& Mullan, D. 2013, MNRAS, 438, 2371

Rodríguez-López, C., MacDonald, J., \& Moya, A. 2012, MNRAS, 419, 44

Sanchis-Ojeda, R., Rappaport, S., Winn, J. N., et al. 2013, ApJ, 774, 54

Skumanich, A. 1972, ApJ, 171, 565

Smith, M. A. 1979, PASP, 91, 737

Smith, J. C., Stumpe, M. C., Van Cleve, J. E., et al. 2012, PASP, 124, 1000

Stumpe, M. C., Smith, J. C., Van Cleve, J. E., et al. 2012, PASP, 124, 985

Swift, J. J., Johnson, J. A., Morton, T. D., et al. 2013, ApJ, 764, 105

Torres, G., Anderson, J., \& Giménez, A. 2010, A\&ARv, 18, 67

Torres, G., \& Ribas, I. 2002, ApJ, 567, 1140

Verbunt, F., \& Zwaan, C. 1981, A\&A, 100, L7

Vida, K., \& Oláh, K. 2013, arXiv:1308.2641

Vida, K., Oláh, K., Kővári, Zs., et al. 2009, A\&A, 504, 1021

Vida, K., Oláh, K., \& Szabó, R. 2014, MNRAS, in press (arXiv:1404.4359)

Wizinowich, P., Acton, D. S., \& Shelton, A. H. 2000, PASP, 112, 315

Wizinowich, P. L., Le Mignant, D., \& Bouchez, C. 2006, PASP, 118, 297

Zwaan, C. 1981, in Solar Phenomena in Stars and Stellar Systems, ed. R. M. Bonnet \& A. K. Dupree (Dordrecht: Reidel), 463 